\title{
The Factory and the Beehive. III. PTFEB132.707+19.810, A Low-mass Eclipsing Binary in Praesepe Observed by PTF and $K 2$
}

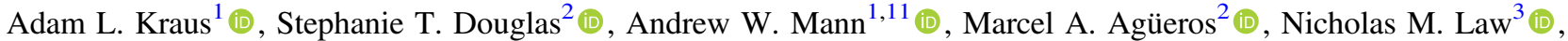

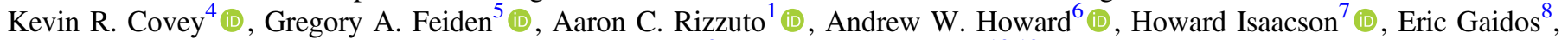 \\ Guillermo Torres $^{9}$ (1), and Gaspar Bakos ${ }^{10,12}$ (B) \\ ${ }^{1}$ Department of Astronomy, The University of Texas at Austin, Austin, TX 78712, USA \\ ${ }^{2}$ Columbia University, Department of Astronomy, 550 West 120th Street, New York, NY 10027, USA \\ ${ }^{3}$ Department of Physics and Astronomy, University of North Carolina, Chapel Hill, NC 27599, USA \\ ${ }^{4}$ Western Washington University, Department of Physics \& Astronomy, Bellingham, WA 98225, USA \\ 5 Department of Physics, University of North Georgia, Dahlonega, GA 30597, USA \\ ${ }^{6}$ California Institute of Technology, 1200 E. California Boulevard, Pasadena, CA 91125, USA \\ ${ }^{7}$ University of California, Berkeley, Berkeley, CA 94720, USA \\ ${ }^{8}$ Department of Geology and Geophysics, University of Hawaii at Manoa, Honolulu, HI 96822, USA \\ ${ }^{9}$ Harvard-Smithsonian Center for Astrophysics, 60 Garden Street, Cambridge, MA 02138, USA \\ ${ }^{10}$ Department of Astrophysical Sciences, Princeton University, Princeton, NJ 08544, USA \\ Received 2017 March 23; revised 2017 June 19; accepted 2017 June 28; published 2017 August 11
}

\begin{abstract}
Theoretical models of stars constitute the fundamental bedrock upon which much of astrophysics is built, but large swaths of model parameter space remain uncalibrated by observations. The best calibrators are eclipsing binaries in clusters, allowing measurement of masses, radii, luminosities, and temperatures for stars of known metallicity and age. We present the discovery and detailed characterization of PTFEB132.707+19.810, a $P=6.0$ day eclipsing binary in the Praesepe cluster $(\tau \sim 600-800 \mathrm{Myr} ;[\mathrm{Fe} / \mathrm{H}]=0.14 \pm 0.04)$. The system contains two late-type stars $\left(\mathrm{SpT}_{P}=\mathrm{M} 3.5 \pm 0.2 ; \mathrm{SpT}_{S}=\mathrm{M} 4.3 \pm 0.7\right)$ with precise masses $\left(M_{p}=0.3953 \pm 0.0020 M_{\odot} ; M_{s}=0.2098 \pm\right.$ $\left.0.0014 M_{\odot}\right)$ and radii $\left(R_{p}=0.363 \pm 0.008 R_{\odot} ; R_{s}=0.272 \pm 0.012 R_{\odot}\right)$. Neither star meets the predictions of stellar evolutionary models. The primary has the expected radius but is cooler and less luminous, while the secondary has the expected luminosity but is cooler and substantially larger (by $20 \%$ ). The system is not tidally locked or circularized. Exploiting a fortuitous 4:5 commensurability between $P_{\text {orb }}$ and $P_{\text {rot,prim }}$, we demonstrate that fitting errors from the unknown spot configuration only change the inferred radii by $\lesssim 1 \%-2 \%$. We also analyze subsets of data to test the robustness of radius measurements; the radius sum is more robust to systematic errors and preferable for model comparisons. We also test plausible changes in limb darkening and find corresponding uncertainties of $\sim 1 \%$. Finally, we validate our pipeline using extant data for GU Boo, finding that our independent results match previous radii to within the mutual uncertainties $(2 \%-3 \%)$. We therefore suggest that the substantial discrepancies are astrophysical; since they are larger than those for old field stars, they may be tied to the intermediate age of PTFEB132.707+19.810.
\end{abstract}

Key words: binaries: eclipsing - stars: evolution - stars: fundamental parameters - stars: individual

(PTFEB132.707+19.810) - stars: low-mass - starspots

Supporting material: machine-readable tables

\section{Introduction}

The fundamental properties of stars establish the foundation for much of astrophysics, and uncertainties in model-derived stellar properties propagate into systematic uncertainties in the initial mass function (Bastian et al. 2010), the age-activity-rotation relation (Agüeros et al. 2011; Douglas et al. 2016; Rebull et al. 2016), and exoplanet masses and radii (e.g., Gaidos \& Mann 2012; Bastien et al. 2014). The relations between mass, radius, luminosity, temperature, and metallicity, or subsets thereof, have traditionally been calibrated with observations of the Sun, stellar populations, or binary systems. However, these calibrations remain sparse and observationally expensive for low-mass stars due to their intrinsically low luminosities. The past 15 years have seen benchmark calibrations for the relations for mass-luminosity (with visual binaries; Delfosse et al. 2000), luminosity-temperatureradius (with long-baseline interferometry; Boyajian et al. 2012), and

\footnotetext{
11 Hubble Fellow.

12 Packard Fellow.
}

luminosity-temperature-metallicity (with spectroscopy; Mann et al. 2015 ), typically with a scatter of $\sim 5 \%$ for mass or radius measurements.

Direct measurements of the mass-radius relation are typically derived from studies of field eclipsing binary systems (e.g., Torres et al. 2010), for which the orbit yields masses and the eclipses yield radii. Eclipsing binaries can yield mass and radius measurements with formal uncertainties of $\lesssim 1 \%$, surpassing the precision offered by visual binaries or longbaseline interferometry, but due to their faintness only a modest number of systems with low-mass components $\left(M_{p} \lesssim 0.7 M_{\odot}\right)$ have been discovered and characterized. The systems discovered to date have suggested that models and observations are discrepant in at least some cases, with models predicting radii that are up to $10 \%$ too low for a given mass (e.g., LópezMorales 2007; Torres et al. 2010). This problem has been seen for solar-type stars for several decades (e.g., Popper 1997; Torres et al. 2006; Torres et al. 2008; Clausen et al. 2009). The most recent compilations by Dittmann et al. (2017) and 
Iglesias-Marzoa et al. (2017) show that this scatter exists for the entire mass range $0.2 M_{\odot}<M<1.0 M_{\odot}$; some stellar radii match model predictions, but most stars are larger.

It remains unclear whether the radius discrepancy results from physics common to all low-mass stellar interiors (e.g., requiring extra opacities or modified treatments of convection, metallicity, or magnetic fields; Feiden \& Chaboyer 2014a, 2014b) or a systematic effect specific to the short-period binary systems that are most likely to eclipse. These short-period systems are often tidally locked to rapid rotation periods that sustain high levels of magnetic activity, possibly leading to larger radii than are predicted by nonmagnetic stellar models (Chabrier et al. 2007; Morales et al. 2009; Kraus et al. 2011; Mullan \& MacDonald 2001). However, even some very long-period systems are larger than models would predict (Irwin et al. 2011).

Eclipsing binaries in star clusters offer even stronger tests, allowing measurement of the masses, radii, luminosities, temperatures, metallicities, and ages of the component stars, but those systems have been rare to date. PTFEB132.707 +19.810-also AD 3814 (Adams et al. 2002), 2MASS J08504984+1948364 (Cutri et al. 2003), and EPIC 211972086 (Huber et al. 2016) — was first suggested as a candidate member of the Praesepe open cluster by Adams et al. (2002), who assessed a membership probability of $48.4 \%$ based on its 2MASS/POSS colors and proper motion. The membership probability was subsequently revised upward to $97.9 \%$ by Kraus \& Hillenbrand (2007) based on its 2MASS/USNO-B1.0/SDSS photometry and proper motion, while Boudreault et al. (2012) assessed a membership probability of $86 \%$ based on UKIDSS photometry and proper motion. Kraus \& Hillenbrand (2007) estimated the spectral type to be M3.4 based on the broadband optical/NIR SED, and West et al. (2011) analyzed the SDSS spectrum to estimate a spectral type of M5.

PTFEB132.707+19.810 was closely inspected by the Palomar Transient Factory (PTF) Open Cluster Survey (POCS) due to its likely Praesepe membership and its nature as a midM dwarf. Agüeros et al. (2011) measured a rotation period of $P_{\text {rot }}=7.43$ days based on clear periodic brightness variations. As we describe below, PTFEB132.707+19.810 also was identified to be an eclipsing binary with an orbital period of $P_{\text {orb }}=6.0$ days that has not locked its stars into synchronous rotation. The star has otherwise remained anonymous in the literature until this year, when its eclipsing nature was recognized by others studying $K 2$ data in Praesepe (Douglas et al. 2017; Gillen et al. 2017; Rebull et al. 2017). In this paper, we analyze our discovery light curve from PTF, follow-up spectroscopic observations, and the newly released $K 2$ light curve of PTFEB132.707+19.810 in order to measure the stellar properties of this mid-M-type eclipsing binary system and test the predictions of stellar evolutionary models. In Section 2, we describe our observations of this system, and in Section 3, we describe the analysis used to interpret those observations. We summarize the resulting properties for the system in Section 4, as well as testing the robustness of our results. Finally, in Section 5, we discuss the implications of our results for the current generation of stellar evolutionary models.

\section{Observations}

\subsection{PTF Photometry}

The PTF uses wide-field photometric observations from the robotic 48 inch Samuel Oschin Telescope (hereafter P48), a
Schmidt camera with a wide focal plane. When PTFEB132.707 +19.810 was observed in 2010 and 2011, P48 was equipped with the CFH12K mosaic camera that had been installed on the Canada-France-Hawaii Telescope (Rahmer et al. 2008). The camera covered a total field of $8 \mathrm{deg}^{2}$ (with $7.26 \mathrm{deg}^{2}$ of useful area, since one chip was nonfunctional), sampled with a pixel scale of 1 ". The observations that we report were taken in the standard PTF observing mode, which uses $60 \mathrm{~s}$ integrations through a Mould $R$ filter, yielding photometry for all stars down to $m_{R} \sim 20 \mathrm{mag}$ (Ofek et al. 2012). The Praesepe field was typically observed 1-2 times per night as part of the POCS campaign (Agüeros et al. 2011), but it was also observed at a more rapid cadence (every 15 minutes, yielding 15-30 images per night) on some nights as part of the PTF/M-dwarfs campaign (Law et al. 2011; Law et al. 2012). PTFEB132.707 +19.810 was observed 622 times over the course of these two PTF programs.

The construction and analysis of light curves for the POCS and $\mathrm{PTF} / \mathrm{M}$ dwarfs programs were described in more detail by Law et al. (2009, 2012) and Agüeros et al. (2011). To briefly summarize, the images were first calibrated to correct for cross talk, perform bias/overscan subtraction, and divide by a contemporaneous superflat. The data were then processed with SExtractor to measure source photometry, and sources were matched across all epochs. The photometric zero points for each epoch were initially estimated based on SDSS photometry for bright stars in the field, and then the zero point for each epoch was optimized to minimize the median photometric variability of all remaining sources, rejecting apparently variable sources. The pipeline typically achieved a photometric stability of 3-5 mmag over periods of months; the photometric uncertainties for all observations of PTFEB132.707+19.810 were limited by photon noise.

PTFEB132.707+19.810 was identified as a candidate eclipsing binary system using a Box Least Squares algorithm (Kovács et al. 2002) that phased the light curves to all possible periods and searched for a transit- or eclipse-like signature. The identification of eclipses was then visually confirmed.

We list all of the photometric measurements for PTFEB132.707+19.810 in Table 1; in Figure 1, we show the light curve spanning three observing seasons.

\subsection{K2 Photometry}

PTFEB132.707+19.810 was observed as EPIC 211972086 by the Kepler spacecraft during Campaign 5 of its repurposed $K 2$ mission (Howell et al. 2014), for which it was proposed as a target by eight proposals, including ours (GO5095; PI: Agüeros). $K 2$ observed PTFEB132.707+19.810 in longcadence mode $\left(t_{\text {int }}=29.4\right.$ minutes $)$ for 73.9 continuous days spanning 2015 April 27-July 10, yielding 3402 exposures of the $9 \times 8$ pixel postage stamp centered on the target. We show the postage stamp superimposed on SDSS images of the field and one frame from the postage stamp in Figure 2. The $K 2$ data were downloaded from the Mikulski Archive for Space Telescopes as target pixel files, which contain the barycentric corrected observation times, the flux measured in each pixel at each epoch, and quality flags. We omitted the 15 exposures where the quality flag is not zero.

The Kepler spacecraft pointing drifted by $\sim 1$ pixel per $6 \mathrm{hr}$ due to solar pressure, and the spacecraft executed a thruster fire every $6 \mathrm{hr}$ to return to its optimal orientation. The data therefore contain a $6 \mathrm{hr}$ periodicity with amplitude $\pm 0.5 \%$ due to the star 
Table 1

PTF Photometry

\begin{tabular}{|c|c|c|c|}
\hline $\begin{array}{l}\text { Epoch } \\
\text { (HJD-2450000) }\end{array}$ & Phase & $\begin{array}{c}R \\
(\mathrm{mag})\end{array}$ & $\begin{array}{c}\sigma_{R} \\
(\mathrm{mag})\end{array}$ \\
\hline 5229.7300 & 0.6192 & 16.987 & 0.060 \\
\hline 5229.7420 & 0.6212 & 16.910 & 0.038 \\
\hline 5229.7430 & 0.6214 & 16.951 & 0.042 \\
\hline 5229.7490 & 0.6224 & 17.035 & 0.044 \\
\hline 5229.7500 & 0.6226 & 16.923 & 0.048 \\
\hline 5229.7550 & 0.6234 & 16.969 & 0.050 \\
\hline 5229.7570 & 0.6237 & 17.015 & 0.060 \\
\hline 5229.7840 & 0.6282 & 16.919 & 0.052 \\
\hline 5229.7980 & 0.6305 & 16.992 & 0.054 \\
\hline 5229.8030 & 0.6314 & 17.057 & 0.043 \\
\hline 5229.8050 & 0.6317 & 16.941 & 0.049 \\
\hline 5229.8240 & 0.6349 & 17.029 & 0.045 \\
\hline 5229.8260 & 0.6352 & 16.996 & 0.044 \\
\hline 5239.7080 & 0.2779 & 17.070 & 0.025 \\
\hline 5239.7100 & 0.2782 & 17.100 & 0.024 \\
\hline 5239.7210 & 0.2800 & 17.052 & 0.024 \\
\hline 5239.7230 & 0.2804 & 17.083 & 0.027 \\
\hline 5239.7330 & 0.2820 & 16.985 & 0.037 \\
\hline 5239.7710 & 0.2884 & 17.075 & 0.049 \\
\hline 5239.7730 & 0.2887 & 17.087 & 0.062 \\
\hline 5239.8040 & 0.2938 & 17.040 & 0.024 \\
\hline
\end{tabular}

(This table is available in its entirety in machine-readable form.)

being sampled across the detector pixels (and their response function) differently over time (Vanderburg \& Johnson 2014). The light curve also shows visually obvious sinusoidal periodicity with a period of $P_{\text {rot }} \sim 7.5$ days and amplitude $\pm 3 \%$, as well as clear primary and secondary eclipses with a periodicity of $P_{\text {orb }} \sim 6.0$ days. The primary star flux contributed $\sim 75 \%$ of the total optical flux (Section 2.4), so the origin of the sinusoidal periodicity was most likely rotational modulation due to spots on the primary. We prepared the light curve for eclipse fitting by using the methods described by Douglas et al. (2016) to measure photometry and rectify the stellar and instrumental variability.

We began by computing and subtracting the background in each exposure using an iterative $3 \sigma$ clipped median and then measured the flux of PTFEB132.707+19.810 through a softedged circular aperture with a radius of 4 pixels, yielding a raw light curve. The aperture was centered on the photocenter in each exposure, so it tracked the drift of the target. We then detrended the long-timescale variability using the Python routine supersmoother ${ }^{13}$ (Friedman 1984; Vanderplas \& Willmer 2015) with a high bass-enhancement value $(\alpha=10)$.

We measured the period of the sinusoidal variability using the Lomb-Scargle function in the Python package gatspy (Vanderplas et al. 2016), an implementation of the Fast Fourier Transform (FFT)-based algorithm from Press \& Rybicki (1989). We computed the periodogram power for $3 \times 10^{4}$ periods spanning 0.1-70.8 days and established false-alarm probabilities using nonparametric bootstrap resampling to generate $10^{3}$ simulated light curves. To divide out the sinusoidal variability, we folded the light curve on the most likely period and used supersmoother to produce a smoothed periodic light curve. After iterating this procedure six times to

\footnotetext{
13 https://github.com/jakevdp/supersmoother
}

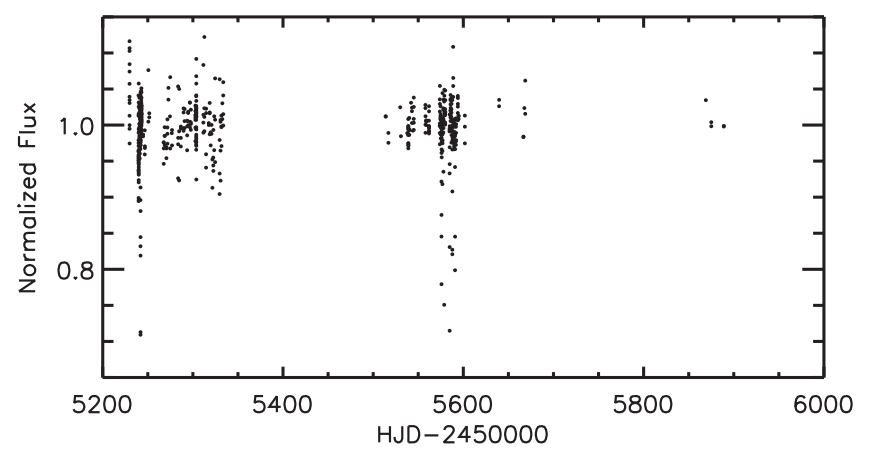

Figure 1. PTF aperture photometry results for the Praesepe eclipsing binary PTFEB132.707+19.810, with fluxes normalized to the median value $\left(m_{R}=17.03 \mathrm{mag}\right)$.

remove stellar variability, we next measured the timedependent flat field at each epoch by calculating the median flux for the 21 other epochs with the closest centroid positions (in detector coordinates). We then returned to the raw light curve and first applied the flat-field correction, then fit and rectified the stellar variability. Finally, we rectified the remaining low-order power in the light curve by dividing the flux at each epoch by the median of all other noneclipse fluxes observed within $\pm 12 \mathrm{hr}$.

We show the stages of this process in Figure 3 and present the resulting normalized fluxes in Table 2. We found a final best-fit rotational period (most likely for the primary) of $P_{\text {rot }}=7.46$ days, which is nearly identical to the period of $P=7.43$ days measured by Agüeros et al. (2011). We also repeated our analysis for the same light curve extracted by K2 Systematics Correction (K2SC; Aigrain et al. 2016) and found a period of $P=7.49$ days. The uncertainties in rotational periods are dominated by systematic effects due to evolution of the (unmeasurable) spot configuration, but different surveys of rotation in open clusters have typically yielded values that agree to within $\lesssim 2 \%-3 \%$ (e.g., Douglas et al. 2016), so these measurements are statistically indistinguishable. The light curve also contains numerous flares; one primary eclipse (at epoch 2361) and one secondary eclipse (at epoch 2364) were contaminated by flares, so we have omitted those observations from our light-curve fits.

\subsection{Archival Photometry}

As part of our analysis to compute a bolometric flux for this system, we also have compiled all of the available (componentunresolved) photometry in all-sky surveys. As we summarize in Table 3, we have used photometry from $\operatorname{SDSS-DR9~}(u, g, r$, $i, z$; Ahn et al. 2012), 2MASS ( $J, H, K_{s}$; Cutri et al. 2003), and AllWISE (W1, W2, W3; Cutri et al. 2013).

\subsection{Keck/HIRES High-resolution Spectroscopy and Radial Velocities}

After our discovery of eclipses in this system in 2010, we began a spectroscopic monitoring campaign to measure radial velocities (RVs) for the components. We obtained 20 highdispersion spectra for the system using Keck I and the HIRES spectrograph (Vogt et al. 1994), which is a single-slit echelle spectrograph permanently mounted on the Nasmyth platform. Ten spectra were obtained using classical observing on three nights. These observations were performed using the red channel and $\mathrm{C} 1$ decker and spanned a wavelength range of 

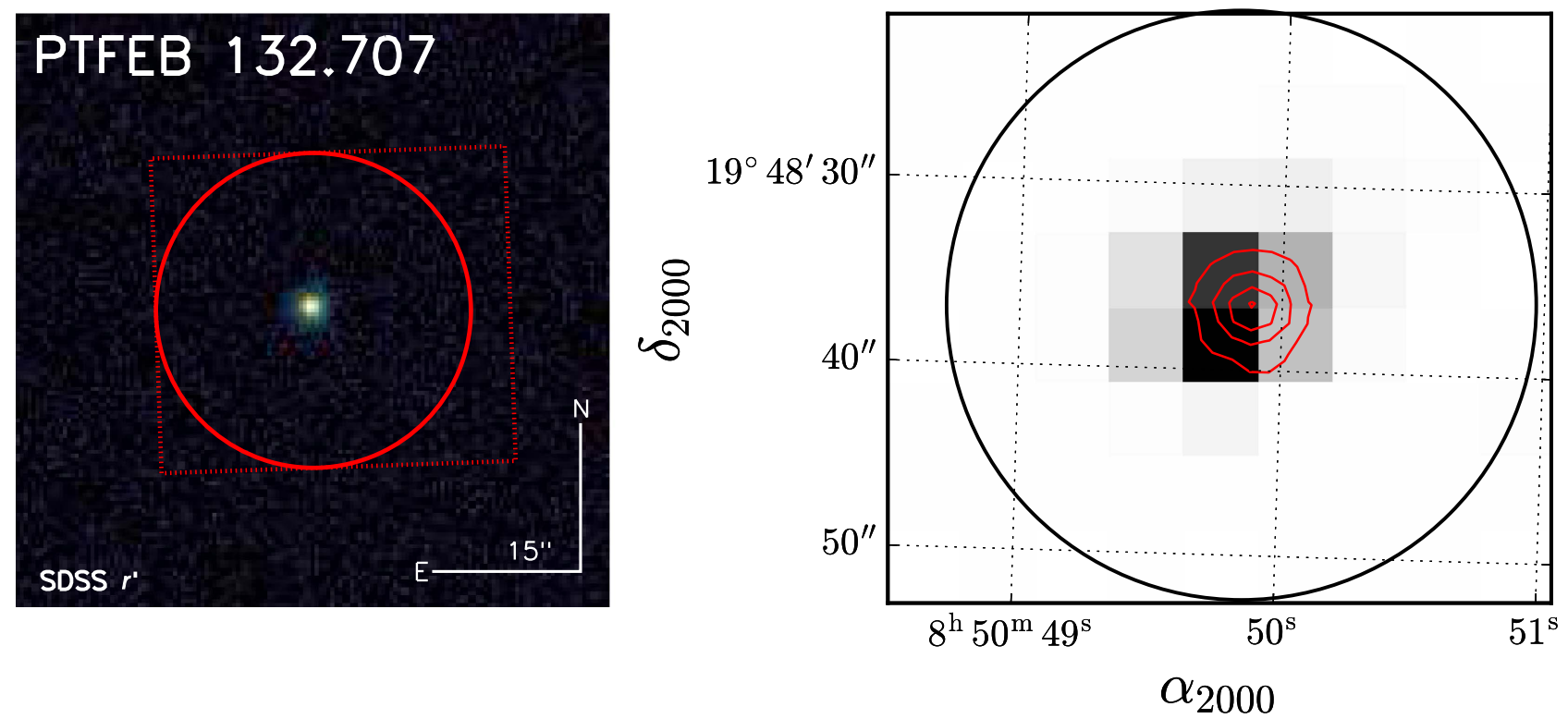

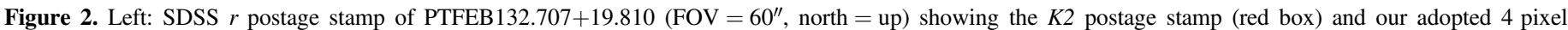

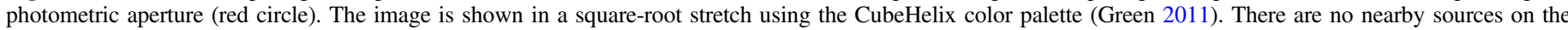

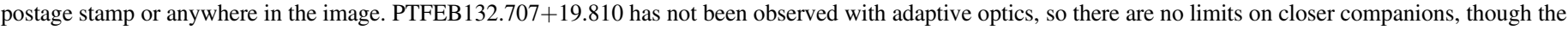

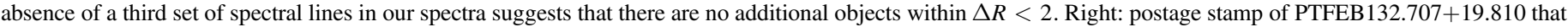

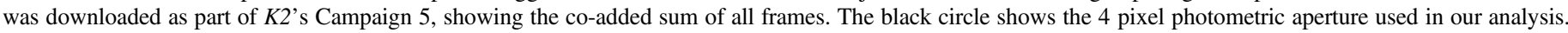
The red contours show the flux distribution of the SDSS image.

$4500-8900 \AA$, yielding a spectral resolution of $R \sim 48,000$. We processed our HIRES data using the standard pipeline MAKEE $^{14}$, which automatically extracts, flat-fields, and wavelength-calibrates spectra taken in most standard HIRES configurations. Ten additional spectra were obtained in a queue mode via collaboration with the California Planet Search (CPS) on nine nights, so as to obtain broader phase coverage for the system. These observations were performed using the red channel and $\mathrm{C} 2$ decker and spanned a wavelength range of 3400-8100 A, also yielding $R \sim 48,000$. These data were extracted using the standard CPS pipeline (Howard et al. 2010). In both cases, we refined the wavelength solution by cross correlating the $7600 \AA^{\mathrm{O}_{2}}$ telluric absorption band against that of the spectrophotometric standard star HZ 44 (Massey et al. 1988).

Our analysis of the HIRES data to measure RVs is identical to the methods described in Kraus et al. (2011, 2014, 2015). For each spectrum, we measured the broadening function (Rucinski 1999) ${ }^{15}$ with respect to three Keck/HIRES observations of two standard stars with similar temperature and metallicity: one observation of Gl 447 and two separate observations of Gl 83.1 on different nights. The broadening function is a better representation of the rotational broadening convolution than a cross correlation, since it is less subject to "peak pulling" and produces a flatter continuum. We fit each broadening function with two Gaussian functions to determine the absolute primary- and secondary-star RVs $\left(v_{p}\right.$ and $\left.v_{s}\right)$, the standard deviations of the line width due to rotation and instrumental resolution $\left(\sigma_{v_{p}}\right.$ and $\left.\sigma_{v_{s}}\right)$, and the average flux ratio across all well-fit orders (which is estimated from the ratio of areas for the two peaks of the broadening function). We list these measurements in Table 4.

\footnotetext{
14 http://www.astro.caltech.edu/ tb/makee/

15 http://www.astro.utoronto.ca/ rucinski/SVDcookbook.html
}

In Table 5, we list the equivalent widths of $\mathrm{H} \alpha$ emission for those epochs where the lines from the two stars were fully resolved (i.e., within $\Delta \phi<0.1$ of orbital quadrature). The equivalent widths are measured with respect to the continuum of the full composite spectrum, but individual stellar values can be determined from the flux ratio of the spectra (which is nearly constant across the entire wavelength range of the HIRES spectra). We also show narrow wavelength ranges around $\mathrm{H} \alpha$ in each spectrum in Figure 4. As can be seen, while the $\mathrm{H} \alpha$ emission of the primary is clearly evident, the emission from the secondary is weaker and can only be measured in aggregate by measuring the excess flux over the continuum within $\pm 1 \AA$ of the expected line center.

To estimate $v \sin (i)$ from $\sigma_{v}$, we artificially broadened each of our template spectra using the IDL task $l s f_{-}$rotate (Gray 1992; Hubeny \& Lanz 2011) using a range of rotational velocities and then computed each artificial spectrum's broadening function using the corresponding original template. This process yielded a relation between $v \sin (i)$ and $\sigma_{v}$ that is appropriate for any spectrum observed with Keck/HIRES at this spectral resolution and can be applied to the $\sigma_{v}$ values that emerge from the Gaussian function that we fit to each component's broadening function peak in our analysis. Using the 10 spectra with the lowest inter-order scatter of $\sigma_{v}$, we find that the mean and standard error of the line broadening for each star are $\sigma_{v, p}=7.78 \pm 0.02 \mathrm{~km} \mathrm{~s}^{-1}$ and $\sigma_{v, s}=7.55 \pm 0.05 \mathrm{~km} \mathrm{~s}^{-1}$, which correspond to $v_{p} \sin (i)=$ $2.6 \pm 0.6 \mathrm{~km} \mathrm{~s}^{-1}$ and $v_{s} \sin (i)<2.0 \mathrm{~km} \mathrm{~s}^{-1}$, respectively.

In Figure 5, we show the flux ratio measured for the two stars, as computed from the broadening functions for each order of each spectrum, as well as the mean and standard error for each order after applying a $2 \sigma$ clip. The flux ratio should depend on wavelength for two stars with unequal temperatures, and we find that there is a positive linear correlation with slope $a=2.97 \times 10^{-5} \AA^{-1}$. As we discuss below, the points constituting this wavelength-dependent relation provide an 


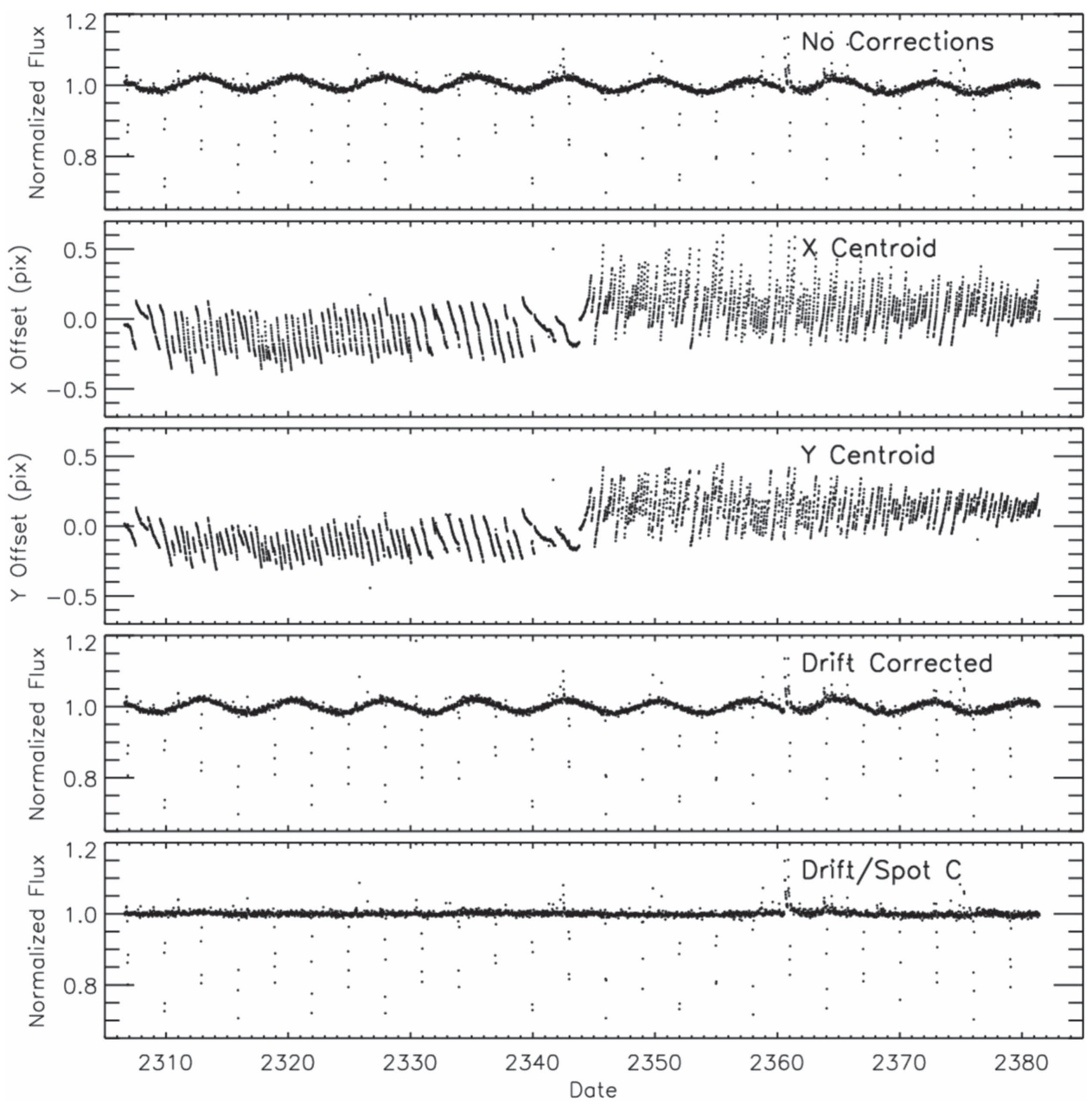

Figure 3. $K 2$ aperture photometry results for the Praesepe eclipsing binary PTFEB132.707+19.810 (EPIC 211972086). Time is specified in units of BJD-2454833, the standard time system for $K 2$. First panel: normalized light curve extracted from aperture photometry, without any subsequent detrending. Second and third panels: $\mathrm{X}$ and $\mathrm{Y}$ centroid positions, in pixels, as a function of time. The $6 \mathrm{hr}$ interval between thruster firings (which reset the telescope position) is evident in the positions, and the position information can be used to detrend flux variations as the target moves across the detector. Fourth panel: normalized light curve after correcting flatfield variations due to telescope drift. Fifth panel: normalized light curve after detrending the phase-folded mean stellar variability due to spots; this light curve is used for eclipse fitting.

important constraint in deconvolving the combined-light spectra taken with the Supernova Integral Field Spectrograph (SNIFS) and SpeX.

\subsection{Intermediate-resolution Spectroscopy}

We obtained an optical spectrum of PTFEB132.707+19.810 on 2016 April 3 with SNIFS (Aldering et al. 2002; Lantz et al. 2004 ) on the University of Hawai' $i 2.2 \mathrm{~m}$ telescope on Maunakea. SNIFS covers $3200-9700 \AA$ simultaneously with a resolution of $R \sim 700$ and $R \sim 1000$ in the blue (3200-5200 $\AA$ ) and red (5100-9700 $\AA$ ) channels, respectively. We observed with a total integration time of $3 \times 1200=3600 \mathrm{~s}$, yielding a signalto-noise ratio $(\mathrm{S} / \mathrm{N})=100$ per resolving element at $\lambda=6500 \AA$. We also observed spectrophotometric standards for flux calibration and obtained a ThAr arc before or after each observation for wavelength calibration. Bias subtraction, flat fielding, dark correction, cosmic-ray rejection, construction of data cubes, and extraction of the final spectrum were performed as described in detail by Aldering et al. (2002). The flux calibration was derived from the combination of the spectrophotometric standards and a model of the atmospheric absorption above Maunakea as described by Mann et al. (2015).

We obtained a NIR spectrum of PTFEB132.707+19.810 on 2016 April 5 with the SpeX spectrograph (Rayner et al. 2003) on the NASA Infrared Telescope Facility on Maunakea. SpeX observations were taken in the short cross-dispersed mode using the 0 " $3 \times 15^{\prime \prime}$ slit, yielding simultaneous coverage from 0.8 to $2.4 \mu \mathrm{m}$ at a resolution of $R \sim 2000$. The target was observed in an $\mathrm{AB}$ nod pattern to allow for sky subtraction. We took a total of 34 exposures totaling $4080 \mathrm{~s}$, yielding $\mathrm{S} / \mathrm{N}=100$ per resolving element at $\lambda=2.2 \mu \mathrm{m}$. Spectra were extracted using the SpeXTool package (Cushing et al. 2004), which performs flat fielding, wavelength calibration, sky subtraction, and extraction of the final spectrum. Exposures were combined using the IDL routine xcombspec. Telluric lines were corrected using a spectrum of the A-type star HD 68703, which was observed immediately before the target with a difference of $<0.1$ airmass, and the correction was computed and applied using the xtellcor package (Vacca et al. 2003).

Following the method outlined by Mann et al. (2015), we combined and absolutely flux calibrated the optical and NIR spectra using published photometry (Section 2.5) with the filter 
Table 2

K2 Photometry

\begin{tabular}{lccc}
\hline \hline $\begin{array}{l}\text { Epoch } \\
\text { (HJD-2450000) }\end{array}$ & Phase & $\begin{array}{c}F \\
\text { (Normalized) }\end{array}$ & $\begin{array}{c}\sigma_{F} \\
\text { (Normalized) }\end{array}$ \\
\hline 7139.6011 & 0.0981 & 0.995 & 0.008 \\
7139.6215 & 0.1015 & 0.999 & 0.007 \\
7139.6419 & 0.1049 & 0.995 & 0.007 \\
7139.6624 & 0.1083 & 0.994 & 0.007 \\
7139.6828 & 0.1117 & 1.002 & 0.007 \\
7139.7032 & 0.1151 & 0.998 & 0.007 \\
7139.7236 & 0.1185 & 1.001 & 0.007 \\
7139.7441 & 0.1219 & 1.015 & 0.007 \\
7139.7645 & 0.1253 & 1.018 & 0.007 \\
7139.7849 & 0.1287 & 1.004 & 0.007 \\
7139.8054 & 0.1321 & 0.996 & 0.007 \\
7139.8258 & 0.1355 & 0.977 & 0.007 \\
7139.8462 & 0.1389 & 0.860 & 0.007 \\
7139.8667 & 0.1423 & 0.799 & 0.007 \\
7139.8871 & 0.1456 & 0.882 & 0.007 \\
7139.9075 & 0.1490 & 0.993 & 0.007 \\
7139.9280 & 0.1524 & 1.000 & 0.007 \\
7139.9484 & 0.1558 & 0.998 & 0.007 \\
7139.9688 & 0.1592 & 0.995 & 0.007 \\
7139.9893 & 0.1626 & 1.000 & 0.007 \\
7140.0097 & 0.1660 & 0.998 & 0.007 \\
\hline
\end{tabular}

(This table is available in its entirety in machine-readable form.)

Table 3

System Photometry

\begin{tabular}{lcl}
\hline \hline Filter & $m(\mathrm{mag})$ & Reference \\
\hline$u$ & $21.021 \pm 0.080$ & SDSS-DR9 (Ahn et al. 2012) \\
$g$ & $18.771 \pm 0.008$ & SDSS-DR9 (Ahn et al. 2012) \\
$r$ & $17.302 \pm 0.006$ & SDSS-DR9 (Ahn et al. 2012) \\
$i$ & $15.807 \pm 0.004$ & SDSS-DR9 (Ahn et al. 2012) \\
$z$ & $14.999 \pm 0.005$ & SDSS-DR9 (Ahn et al. 2012) \\
$J$ & $13.529 \pm 0.026$ & 2MASS (Cutri et al. 2003) \\
$H$ & $12.911 \pm 0.024$ & 2MASS (Cutri et al. 2003) \\
$K_{s}$ & $12.651 \pm 0.022$ & 2MASS (Cutri et al. 2003) \\
$W 1$ & $12.497 \pm 0.024$ & ALLWISE (Cutri et al. 2013) \\
$W 2$ & $12.330 \pm 0.024$ & ALLWISE (Cutri et al. 2013) \\
\hline
\end{tabular}

profiles and zero points provided by Fukugita et al. (1996) and Mann \& von Braun (2015).

\section{Analysis}

\subsection{Atmospheric Properties and Radius Ratio from Spectra}

We initially analyzed the system as a single, unresolved object. Following Mann et al. (2015), we combined the optical and NIR spectra (Section 2.5), which we simultaneously flux calibrated using available photometric measurements (Section 2.3) and the appropriate zero points and filter profiles (Cohen et al. 2003; Mann \& von Braun 2015). We filled in missing regions of the spectrum and areas of high telluric contamination with the best-fit BT-SETTL atmospheric model (Allard et al. 2011). Once combined and calibrated, we dereddened the spectrum by $E(B-V)=0.027 \pm 0.004 \mathrm{mag}$ (Taylor 2006) and then integrated over wavelength to compute the bolometric flux. Accounting for errors in the flux calibration, photometry, photometric zero points, and reddening, we derived a final bolometric flux of $F_{\mathrm{bol}}=(1.75 \pm 0.06) \times$ $10^{-11} \mathrm{erg} \mathrm{cm}^{-2} \mathrm{~s}^{-1}$. To compute the bolometric luminosity, we adopted the distance $d=182 \pm 6 \mathrm{pc}$ (van Leeuwen 2009) and found $L_{\mathrm{bol}}=0.0180 \pm 0.0010 L_{\odot}$.

The luminosities and temperatures of the individual stars are subject to a strong joint constraint from the unresolved magnitudes and spectra of the PTFEB132.707+19.810 system when combined with Praesepe's known distance and reddening. However, the colors and molecular absorption bands of M dwarfs vary smoothly and monotonically with temperature, so the same unresolved features are degenerately consistent with a range of plausible temperature and luminosity combinations. To determine nondegenerate temperatures and luminosities for each star, we therefore must also use the wavelength-dependent flux ratio inferred from our Keck/ HIRES observations (Section 2.4; Figure 5). The same analysis also provides a useful prior for our light-curve analysis. While eclipse light curves strongly constrain the sum of the stellar radii, they offer a weaker constraint on the ratio of the radii; a very similar light curve results from making one star larger and the other smaller and then optimizing the inclination to match.

We have combined all of these data in a simultaneous fit against a library of empirical, flux-calibrated spectra. We adopted these library spectra from the large sample of nearby M dwarfs considered by Mann et al. (2015). These stars have high-quality measurements of their distances $d$ (from parallax), metallicities $[\mathrm{Fe} / \mathrm{H}]$ (from spectra; Mann et al. 2013b), bolometric fluxes $F_{\text {bol }}$ (from spectra and panchromatic broadband photometry), and effective temperatures $T_{\text {eff }}$ (from colors and spectra, using a relation bootstrapped from stars with interferometric radius measurements; Boyajian et al. 2012; Mann et al. 2013a). Using the Stefan-Boltzmann law and the known values of $d, F_{\mathrm{bol}}$, and $T_{\mathrm{eff}}$, we computed the radius of each library star. We then combined it with the absolute fluxcalibrated spectra to compute the emergent spectral flux density or surface brightness $\left(S_{\lambda}\right.$, in $\left.\operatorname{erg~s}^{-1} \mathrm{~cm}^{-2} \AA^{-1}\right)$ of the star, as well as the emergent spectral flux densities when averaged across each order of our HIRES spectra and when convolved with the Kepler and PTF bandpasses ( $S_{K e p}$ and $\left.S_{\mathrm{PTF}}\right)$.

After constructing this library, we then combined all possible pairs of templates with metallicities consistent with Praesepe $([\mathrm{Fe} / \mathrm{H}]=0.14 \pm 0.04$; Taylor 2006) and compared them to the absolutely calibrated unresolved spectrum (Section 2.5) and spectrally resolved HIRES flux ratios (Section 2.4) of the PTFEB132.707+19.810 A+B system. For each system, our analysis explored the range of allowed total flux ratios (and hence radius and surface brightness ratios) that was consistent with the Keck/HIRES and SNIFS+SpeX results. For each possible combination, we solved for the component stellar radii that would best reproduce the absolute and relative flux measurements of PTFEB132.707+19.810 and adjusted the total brightness of the template spectra as appropriate. From the scaled spectra, we computed the radius ratio $\left(\frac{R_{s}}{R_{p}}\right)$, Kepler bandpass flux ratio $\left(\frac{S_{s, \text { Kep }}}{S_{p, \text { Kep }}}\right)$, PTF bandpass flux ratio $\left(\frac{S_{\text {s,PTF }}}{S_{p, \text { PTF }}}\right)$, and $\chi^{2}$ of the fit as dependent variables. We show one example of this fitting procedure in Figure 6, representing the sum of templates that produce the best fit.

The $\chi^{2}$ goodness-of-fit statistic is poorly defined for spectra that have a very large number of wavelength channels and errors that are dominated by the covariance between channels. These covariances can be integrated into the analysis using tools such as Starfish (Czekala et al. 2015), but the run time for a large spectral library would be infeasibly long. To avoid 
Table 4

Keck I/HIRES RVs

\begin{tabular}{|c|c|c|c|c|c|c|c|c|}
\hline $\begin{array}{l}\text { Target/ } \\
\text { Epoch }^{\mathrm{a}}\end{array}$ & $\begin{array}{c}\text { Epoch } \\
\text { (HJD-2450000) }\end{array}$ & $\begin{array}{l}\text { Wavelength } \\
\text { Range }(\AA)\end{array}$ & $\begin{array}{l}t_{\text {int }} \\
(\mathrm{s})\end{array}$ & $\begin{array}{c}v_{p} \\
\left(\mathrm{~km} \mathrm{~s}^{-1}\right)\end{array}$ & $\begin{array}{c}\sigma_{v_{p}}^{\mathrm{b}} \\
\left(\mathrm{km} \mathrm{s}^{-1}\right)\end{array}$ & $\begin{array}{c}v_{s} \\
\left(\mathrm{~km} \mathrm{~s}^{-1}\right)\end{array}$ & $\begin{array}{c}\sigma_{v_{s}{ }^{\mathrm{b}}}\left(\mathrm{km} \mathrm{s}^{-1}\right)\end{array}$ & $F_{s} / F_{p}$ \\
\hline 20101213.36161 & 5543.92230 & $4450-8910$ & 600 & $1.53 \pm 0.08$ & $7.84 \pm 0.10$ & $96.38 \pm 0.22$ & $7.45 \pm 0.27$ & $0.259 \pm 0.013$ \\
\hline 20101213.46220 & 5544.03873 & $4450-8910$ & 600 & $0.42 \pm 0.09$ & $7.79 \pm 0.07$ & $97.74 \pm 0.23$ & $7.48 \pm 0.24$ & $0.279 \pm 0.011$ \\
\hline 20101213.49879 & 5544.08108 & $4450-8910$ & 450 & $0.29 \pm 0.11$ & $7.77 \pm 0.10$ & $98.72 \pm 0.26$ & $7.50 \pm 0.26$ & $0.274 \pm 0.017$ \\
\hline 20120104.40672 & 5930.97583 & $4450-8910$ & 600 & $44.04 \pm 0.10$ & $7.77 \pm 0.09$ & $16.96 \pm 0.18$ & $7.60 \pm 0.16$ & $0.283 \pm 0.013$ \\
\hline 20120104.46819 & 5931.04698 & $4450-8910$ & 900 & $46.56 \pm 0.10$ & $7.84 \pm 0.08$ & $12.31 \pm 0.14$ & $7.84 \pm 0.22$ & $0.266 \pm 0.012$ \\
\hline 20120106.47033 & 5933.04954 & $4450-8910$ & 1200 & $56.81 \pm 0.10$ & $7.83 \pm 0.06$ & $-6.78 \pm 0.19$ & $7.39 \pm 0.12$ & $0.261 \pm 0.012$ \\
\hline 20120106.55325 & 5933.14552 & $4450-8910$ & 600 & $53.91 \pm 0.12$ & $7.84 \pm 0.07$ & $-2.04 \pm 0.30$ & $7.44 \pm 0.25$ & $0.279 \pm 0.014$ \\
\hline 20100526.23134 & 5342.76526 & $3360-8100$ & 900 & $68.01 \pm 0.19$ & $8.02 \pm 0.14$ & $-31.24 \pm 0.49$ & $8.13 \pm 0.55$ & $0.263 \pm 0.017$ \\
\hline 20100603.22375 & 5350.75579 & $3360-8100$ & 900 & $14.87 \pm 0.11$ & $7.89 \pm 0.13$ & $69.87 \pm 0.34$ & $7.35 \pm 0.48$ & $0.229 \pm 0.023$ \\
\hline 20101121.44093 & 5522.01223 & $3360-8100$ & 450 & $45.15 \pm 1.52$ & $8.92 \pm 0.87$ & $8.88 \pm 0.43$ & $7.72 \pm 0.20$ & $0.291 \pm 0.025$ \\
\hline 20101122.52457 & 5523.10914 & $3360-8100$ & 450 & $67.70 \pm 0.22$ & $7.92 \pm 0.20$ & $-29.52 \pm 0.85$ & $8.68 \pm 0.88$ & $0.312 \pm 0.027$ \\
\hline 20110123.41492 & 5584.98588 & $3360-8100$ & 600 & $26.75 \pm 0.16$ & $7.99 \pm 0.23$ & $45.95 \pm 1.12$ & $6.73 \pm 0.86$ & $0.219 \pm 0.041$ \\
\hline 20120105.39850 & 5931.96637 & $3360-8100$ & 600 & $66.37 \pm 0.13$ & $7.82 \pm 0.11$ & $-28.96 \pm 0.42$ & $7.41 \pm 0.28$ & $0.276 \pm 0.016$ \\
\hline Gl 83.1 & 5741.13595 & $4320-8750$ & 120 & $\cdots$ & $\cdots$ & $\cdots$ & $\ldots$ & $\cdots$ \\
\hline Gl 83.1 & 5930.69346 & $4320-8750$ & 120 & $\cdots$ & $\ldots$ & $\ldots$ & $\cdots$ & $\cdots$ \\
\hline Gl 447 & 5933.16968 & $4320-8750$ & 120 & $\cdots$ & $\ldots$ & $\cdots$ & $\cdots$ & $\ldots$ \\
\hline $\mathrm{HZ} 44$ & 5931.18067 & $4320-8750$ & 120 & $\ldots$ & $\ldots$ & $\ldots$ & $\cdots$ & $\cdots$ \\
\hline
\end{tabular}

Notes. In each observation, the component velocities are subject to a shared systematic uncertainty of $\pm 300 \mathrm{~m} \mathrm{~s}^{-1}$ from the uncertainty in the absolute RV scale. Furthermore, the velocities at all epochs are subject to a shared systematic uncertainty of $\pm 170 \mathrm{~m} \mathrm{~s}^{-1}$ because they are all measured with respect to the same three calibrator stars, each of which has a systematic uncertainty of $\pm 300 \mathrm{~m} \mathrm{~s}^{-1}$.

${ }^{a}$ The first column lists either the UT date and time stamp from the Keck Observatory Archive (for observations of PTFEB132.707+19.810) or the target name (for standard stars).

${ }^{\mathrm{b}}$ We report $\sigma_{v_{p}}$ and $\sigma_{v_{s}}$ as the standard deviation of the Gaussian fits to the two stars' broadening functions, which is a measure of both the instrumental and the rotational broadening. We discuss the conversion to $v \sin (i)$ in Section 2.4.

(This table is available in machine-readable form.)

Table 5

$\mathrm{H} \alpha$ Equivalent Widths Near Quadrature

\begin{tabular}{lccc}
\hline \hline $\begin{array}{l}\text { Epoch } \\
(\text { HJD-2450000) }\end{array}$ & Phase & $\begin{array}{c}\text { EW } \underset{(\mathrm{H} \alpha}{(\mathrm{H})}]_{P} \\
\text { (A) }\end{array}$ & $\begin{array}{c}\mathrm{EW}[\mathrm{H} \alpha]_{S} \\
(\AA)\end{array}$ \\
\hline 55342.765 & 0.414 & 3.09 & 0.50 \\
55350.756 & 0.742 & 3.07 & 1.32 \\
55522.012 & 0.210 & 3.55 & 2.37 \\
55523.109 & 0.392 & 2.70 & 0.52 \\
55542.927 & 0.687 & 3.40 & 2.80 \\
55544.081 & 0.878 & 3.48 & 0.45 \\
55931.966 & 0.357 & 3.27 & 0.45 \\
\hline
\end{tabular}

having the fit dominated by the spatially unresolved spectra, we instead weighted the final $\chi^{2}$ contributions of the unresolved spectrum to equal twice the combined contributions of the HIRES flux ratio (with 27 degrees of freedom) and rescaled all of the $\chi^{2}$ values so that the best-fit value would have $\chi_{\nu}^{2}=1$. Adjusting the weighting by a factor of 5 did not change the results in a significant way.

The result of this analysis is a posterior distribution for the radius ratio $\frac{R_{s}}{R_{p}}$, the Kepler bandpass $\left(K_{p}\right)$ surface brightness ratio $\frac{S_{s, \text { Kep }}}{S_{p, \text { Kep }}}$, and the PTF bandpass (Mould $R$ ) surface brightness ratio $\frac{S_{s, \mathrm{PTF}}}{S_{p, \mathrm{PTF}}}$. There are several confounding variables (such as metallicity, stellar age, and spot coverage), and even small errors in the flux calibration can lead to significant spectral mismatch across many channels, so there is not a smooth $\chi^{2}$ hypersurface within this three-dimensional space. Adjacent points differ significantly in $\chi^{2}$. We instead constructed the joint posterior using the 9276 template pairs with a goodness of fit $\chi_{\nu}^{2}<4$ (chosen to yield visually acceptable matches to the spectra), taking the density of fit results in the threedimensional space as a measure of the posterior probability density. To avoid establishing a prior that goes to zero outside of the distribution of points, but instead declines smoothly away from this locus, we defined the density at a given location in parameter space by convolving each discrete point with a 3D Gaussian blurring function, with $\sigma=0.05$ on all axes. We verified that the shape of this posterior does not change significantly for $\chi^{2}$ cuts or different values of $\sigma$, even extending to much poorer fits $\left(\chi_{\nu}^{2} \sim 10\right)$ where the spectral mismatch is visually obvious. In Figure 7, we show the distribution of 9276 points that defines our posterior distribution for $\frac{R_{s}}{R_{p}}, \frac{S_{s, \text { Kep }}}{S_{p, \text { Kep }}}$, and $\frac{S_{s, \mathrm{PTF}}}{S_{p, \mathrm{PTF}}}$.

In addition to computing a spectroscopic prior for our Markov chain-Monte Carlo (MCMC) orbit analysis, we also used the same template library and fitting scheme to estimate posteriors for the best-fit temperatures, spectral types, and bolometric fluxes for the components of PTFEB132.707 


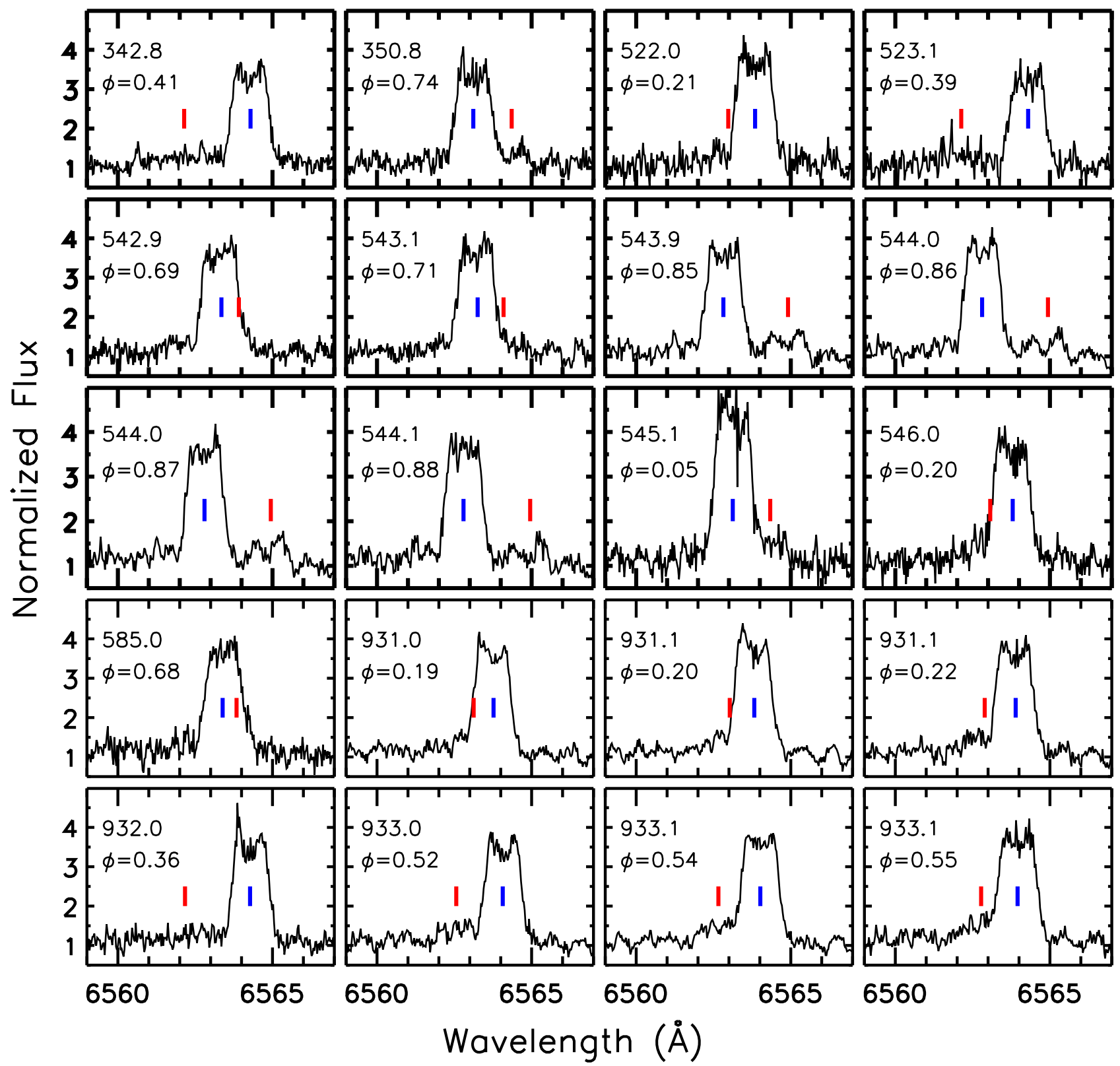

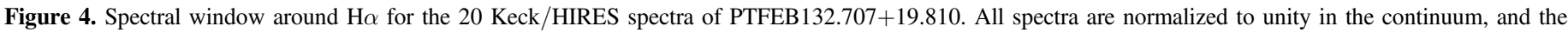

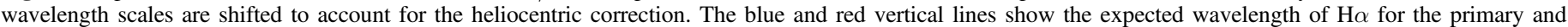

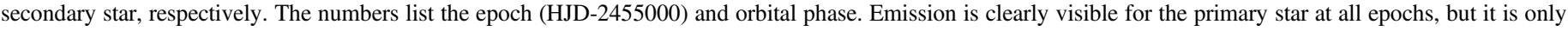

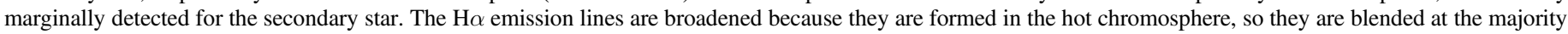
of epochs.

+19.810 . To take advantage of the strong constraints on the surface brightness ratio that emerge from the light curves (Section 3.2), we computed our MCMC analysis of the light curve without using the prior on the surface brightness ratios and radius ratio (to avoid double-weighting the flux ratio constraints from the spectroscopic observations) and then used the resulting posteriors on $\frac{R_{s}}{R_{p}}, \frac{S_{s, \text { Kep }}}{S_{p, \text { Kep }}}$, and $\frac{S_{s, \text { PTF }}}{S_{p, \text { PTF }}}$ as input priors for the analysis described above. We adopted the resulting set of all template pairs with $\chi_{\nu}^{2}<4$ as a posterior distribution for the individual component temperatures, spectral types, bolometric fluxes, and bolometric luminosities. We find that the two template stars that produce the lowest overall $\chi^{2}$ are HD 18143 C $\left(T_{\text {eff }}=3227 \mathrm{~K} ;[\mathrm{Fe} / \mathrm{H}]=+0.28 \pm 0.03\right)$ and GJ 3668 $\left(T_{\text {eff }}=3109 \mathrm{~K} ;[\mathrm{Fe} / \mathrm{H}]=-0.07 \pm 0.08\right)$.

\subsection{MCMC Fitting for Orbital and Stellar Parameters}

We have fit the system properties using an updated version of the MCMC procedure that we described in more detail in our analysis of the low-mass eclipsing binary UScoCTIO 5 (Kraus et al. 2015). To briefly summarize, our pipeline simultaneously fits the RV curve and all available light curves with a model consisting of six orbital elements $\left(T_{0}, P, a, e, \omega\right.$, and $\left.i\right)$, the mass ratio of the system $q=M_{s} / M_{p}$, the systemic RV $\gamma$, the sum of the stellar radii $R_{\mathrm{tot}}=R_{p}+R_{s}$, the ratio of the stellar radii $r=R_{s} / R_{p}$, and the ratios of stellar fluxes through the Kepler $K_{p}$ bandpass and PTF $R$ bandpass.

We fit the RV curves with analytically determined RVs at each epoch; none of our spectra were taken during eclipse, so we do not need to include the Rossiter-McLaughlin effect. We fit the 


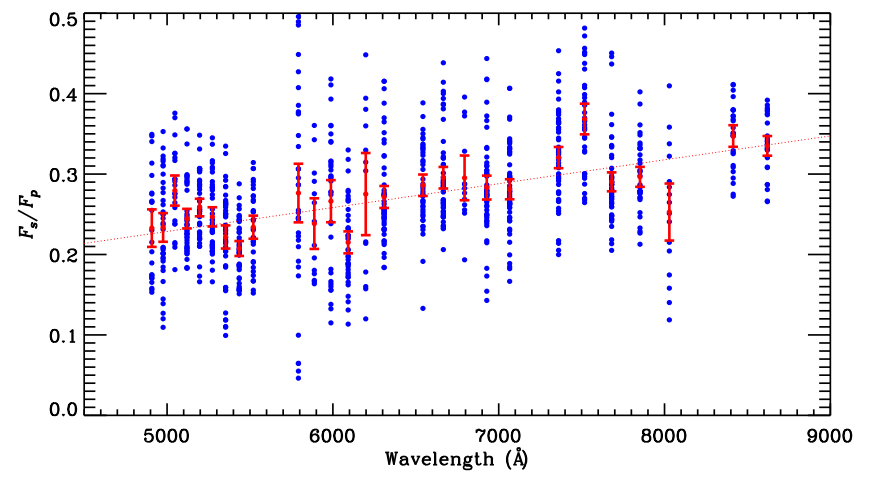

Figure 5. Flux ratio between the binary components as a function of wavelength, as inferred from the ratio of areas under the broadening function peaks. Blue points show individual measurements for each order of each spectrum, with respect to each of the three RV standards. Red points with error bars show the mean and standard error for each order after $\sigma$-clipping outliers with a $2 \sigma$ clip. There is a clear linear trend for the secondary to contribute a larger fraction of the total flux at longer wavelengths: $\frac{F_{s}}{F_{p}}=[2.9709 \times$ $\left.10^{-5} \AA^{-1}\right] \lambda+0.080$. This trend indicates that the secondary is indeed cooler than the primary.

light curves with an analytic formalism based on the work of Mandel \& Agol (2002), with modification to allow for luminous occulting bodies. The $K 2$ exposure time ( 29.4 minutes) is long compared to the typical change in brightness during eclipses, so we modeled 27 subexposures of duration $65.4 \mathrm{~s}$ and then summed those fluxes for comparison to the observations. To model limb darkening, we used a quadratic relation with the coefficients prescribed for a star of appropriate $T_{\text {eff }}$ and $\log (g)$ by Claret et al. (2012): $\quad \gamma_{1, P, R}=0.6171, \quad \gamma_{2, P, R}=0.3327, \quad \gamma_{1, S, R}=0.5436$, $\gamma_{2, S, R}=0.2532, \gamma_{1, P, K p}=0.4930, \gamma_{2, P, K p}=0.4298, \gamma_{1, S, K p}=$ 0.4488 , and $\gamma_{2, S, K p}=0.2818$.

Our algorithm was chosen due to its fast run time, which allows for efficient exploration of our high-dimensional model by our MCMC, but this design choice also comes with necessary caveats. We do not include several physical effects that are modeled in more sophisticated code (e.g., Wilson \& Devinney 1971), such as reflected light and ellipsoidal variation. However, those effects are negligible for mainsequence stars in well-detached systems. More significantly, our code also does not include any model for starspots. As can be seen in Figure 3, the photometric amplitude of the system outside of eclipse $( \pm 3 \%)$ indicates the presence of large and complicated spot complexes. Occultation of those spots will introduce high-frequency noise in the eclipse light curves. Traditionally, these spots are fit with a spot model that is consistent with the out-of-eclipse variations, implicitly rectifying the variations in system flux. However, spatially unresolved photometry does not contain sufficient information to reconstruct a unique distribution of starspots across the stellar surfaces, so the variations are typically modeled with one or two very large spots. These incorrect spot models will degrade the precision of the eclipse fit by simultaneously not encompassing the fine details of the spot structure (which cannot be fit from the variations in total system flux) and forcing the fit to account for a spot model that is not correct. As we discussed in Kraus et al. (2011), uncorrected spots result in radius variations of $\pm 2 \%$; we explore this possibility in further detail in Section 4.3.

We have modified several aspects of our pipeline since our analysis of UScoCTIO 5 in Kraus et al. (2015).
1. Multiple filters: Since we have multiband photometry for PTFEB132.707+19.810 $\left(R_{\mathrm{PTF}}\right.$ and $K_{p}$, albeit not simultaneously), we now fit for a surface brightness ratio in each of these bandpasses.

2. Spectroscopic flux ratios: We previously used the optical flux ratio inferred from the broadening functions of each star in Keck/HIRES spectra (Section 2.4; Figure 5) as a direct constraint on the radius ratio and the $K_{p}$ surface brightness ratio, $\frac{F_{s}}{F_{p}}=\frac{S_{s, \text { Kep }}}{S_{p, \text { Kep }}} \times\left(\frac{R_{s}}{R_{p}}\right)^{2}$. However, this choice did not fully exploit the measurable wavelength dependence of the HIRES flux ratio. We now incorporate the Keck measurements into the analysis of the component temperatures and bolometric fluxes (Section 3.1) and use the posterior joint constraints on $\frac{R_{s}}{R_{p}}, \frac{S_{s, \text { Kep }}}{S_{p, \text { Kep }}}$, and $\frac{S_{s, \mathrm{PTF}}}{S_{p, \mathrm{PTF}}}$ as priors for our MCMC fit of the RV and light $S_{p, \mathrm{PTF}}$
curves.

3. Fitting $T_{P}$ instead of $T_{0}$ : For eclipsing systems that are nearly circular, the combination of the longitude of periastron $\omega$ and the time of periastron $T_{0}$ are highly degenerate and poorly constrained. A Gibbs sampler that separately explores these parameters will mix very slowly due to this degeneracy. We therefore have modified our code to fit the time of primary eclipse $T_{P}$ (which is very well constrained by the eclipse photometry) and $\omega$ and then to compute $T_{0}$ as a dependent quantity. The net result is equivalent to an MCMC that explores on a linear combination of $T_{0}$ and $\omega$ (but without the need to calculate this linearization explicitly for each system) or on $\sqrt{e} \cos (\omega)$ and $\sqrt{e} \sin (\omega)$ (Eastman et al. 2013).

We use a uniform prior for all variables. The eccentricity is not bounded at $e=0$; if a jump reduces the eccentricity to $e<0$, then the eccentricity is made positive and $\omega$ is rotated by $90^{\circ}$. The mass ratio is not bounded at $q=1$, allowing for the star labeled as the secondary to become more massive. If a jump would increase the inclination to $i>90^{\circ}$, then the inclination is set to $180^{\circ}-i$.

We executed the MCMC using a Metropolis-Hastings sampler to walk through parameter space, selecting jump sizes and establishing initial burn-in using test chains from a range of starting parameter states. For the final parameters, we computed 20 simultaneous chains for a total length of $1.1 \times 10^{5}$ steps per chain, omitting the first $10^{4}$ steps of each chain to allow for random dispersal from the (common) initial starting point. As a result, our distributions have $2 \times 10^{6}$ distinct samples from which the posteriors on each parameter are constructed. We verified that the individual chains yield mean values that agree to within much less than the reported $1 \sigma$ uncertainties, indicating that they are well mixed. We also calculated other parameters of interest $\left(M_{p}, M_{s}, M_{\mathrm{tot}}=M_{p}+M_{s}, R_{p}, R_{s}\right)$ from the fit parameters at each step in the chain, yielding similar posterior distributions. Finally, we explored the robustness of our results by fitting many subsets of the data (Section 4.2), where for each subset we computed 20 simultaneous chains for a total length of $2 \times 10^{4}$ steps per chain, omitting the first $10^{4}$ steps for burn-in and dispersion from the initial starting point.

As we discuss further in the Appendix, we have validated our pipeline by analyzing extant data for the well-studied system GU Boo (LMR05), showing that our very different analysis methods match previous radius measurements to within $2 \%-3 \%$. A similar result was found by Windmiller et al. (2010) using the 

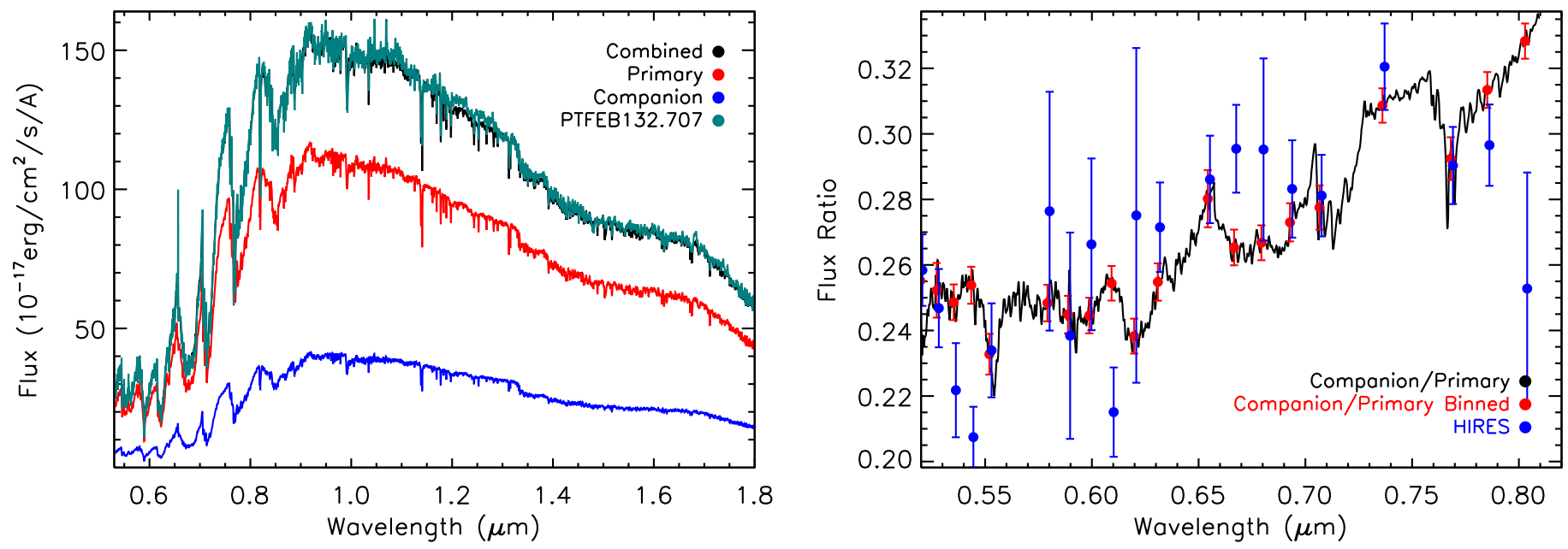

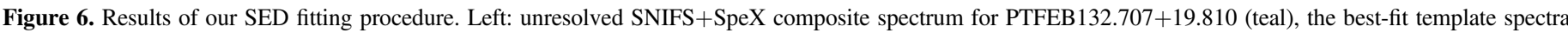

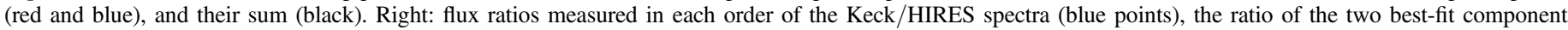
spectra (black line), and the corresponding binned flux ratio values for direct comparison (red points).

ELC software (Orosz \& Hauschildt 2000) and additional data on the GU Boo system.

\section{Results}

\subsection{System Properties}

PTFEB132.707+19.810 is one of the few low-mass $\left(M_{p} \lesssim\right.$ $0.7 M_{\odot}$ ) eclipsing binaries to be found in an open cluster (see, e.g., David et al. 2015, 2016), and therefore it poses a test of main-sequence stellar models for which the metallicity $([\mathrm{Fe} / \mathrm{H}]=$ $0.14 \pm 0.04$; Taylor 2006), distance $(d=182 \pm 6 \mathrm{pc}$; van Leeuwen 2009), and age ( $\tau \sim 600-800 \mathrm{Myr}$; Delorme et al. 2011; Brandt \& Huang 2015) are not confounding free parameters. Furthermore, the long orbital period and lack of tidal locking suggest that the properties of the two stars are broadly representative of typical young zero-age main-sequence (ZAMS) stars, in contrast to the short-period, tidally locked rapid rotators that comprise most of the eclipsing binary sample studied to date.

We summarize our best-fit properties of PTFEB132.707 +19.810 and its components in Table 6 and in Figures 8-10, we show the observed RVs and $K 2$ /PTF photometry, the best-fit model RV and light curves, and the residuals between the observations and the data. We find that PTFEB132.707+19.810 consists of stars with unequal masses $\left(M_{p}=0.395 M_{\odot}\right.$, $\left.M_{s}=0.210 M_{\odot}\right)$ and $\operatorname{radii}\left(R_{p}=0.36 R_{\odot}, R_{s}=0.27 R_{\odot}\right)$. The fractional uncertainties on the individual masses are $\lesssim 1 \%$ due to the dense phase coverage and excellent instrumental stability of the Keck/HIRES data. The fractional uncertainties on the primary and secondary radii are $\sim 2 \%$ and $\sim 4 \%$, respectively. We discuss possible systematic errors in the radius measurements in Sections 4.2, 4.3, and 4.4.

In Figure 11, we show the marginalized one-dimensional posterior distributions of each parameter, as well as the median and central $68 \%$ credible interval. Most posteriors are distributed symmetrically about the median, and therefore the median and central interval are good representations of the most likely value. The clearest exception to this case is the eccentricity distribution, for which the mode of the distribution $(e \sim 0.0013)$ is located just outside the lower edge of the central $68 \%$ credible interval. However, the difference between the median and mode does not change any astrophysically useful results. The other clearly asymmetric distributions are those of $\omega$ and $T_{0}$, which are tightly correlated (Section 3.2), but again, the detailed values do not impact our conclusions.

In Figure 12, we show a triangle plot of the six astrophysically important parameters that are most likely to be degenerate with each other: $e, i, R_{p}+R_{s}, \frac{R_{s}}{R_{p}}, \frac{S_{s}}{S_{p}}$ Kep , and $\frac{S_{s}}{S_{p}}$. The only apparent degeneracies among parameters are those that are well known for eclipse binary analyses. Most significantly, the radius ratio $\frac{R_{s}}{R_{p}}$ is tightly correlated with the inclination, since the fractional occulted area (and hence the depth and duration of the transit) only changes very gradually while changing the impact parameter and the relative stellar radii. However, changing the inclination does change the total sum of the radii that is needed to preserve the eclipse morphology, so there is also a looser correlation between $R_{t o t}$ and both $\frac{R_{s}}{R_{p}}$ and $i$.

The stellar rotation periods can be inferred from both the light curve (for the primary star) and the high-resolution spectra (for both stars and assuming spin-orbit alignment). The light curve is dominated by flux from the primary star, contributing $\sim 75 \%$ of the flux in the red optical (Figure 6). If the observed sinusoidal variations (with full amplitude 6\%) were caused by the secondary star, then its individual total amplitude of variation would be $26 \%$; studies of rotational variability across the full sample by Douglas et al. (2017) and Rebull et al. (2017) found that the maximum amplitude seen for $0.2 M_{\odot}$ stars was only $10 \%$. A similar upper envelope was seen for periodic field stars observed by Kepler by Harrison et al. (2012). We therefore conclude that the photometric variations outside of eclipse (Section 2.2 and Figure 3) show that $P_{\text {rot }, P}=7.46$ days.

Given our measured radius, the corresponding rotational broadening of our spectra would be $v_{p} \sin (i) \sim 2.5 \mathrm{~km} \mathrm{~s}^{-1}$, which is consistent with the measured rotational broadening of $v_{p} \sin (i)=2.6 \pm 0.6 \mathrm{~km} \mathrm{~s}^{-1}$. The rotational signature of the secondary star is not evident in our light curves, but our measured radius and the upper limit on $v \sin (i)$ from our Keck/ HIRES spectra $\left(v_{s} \sin (i)<2.0 \mathrm{~km} \mathrm{~s}^{-1}\right)$ imply a rotational period of $P_{\mathrm{rot}, S} \gtrsim 6$ days. Our measurement would be consistent with tidal locking of the secondary, but we cannot verify whether this has occurred. The rotational period of the $0.4 M_{\odot}$ 

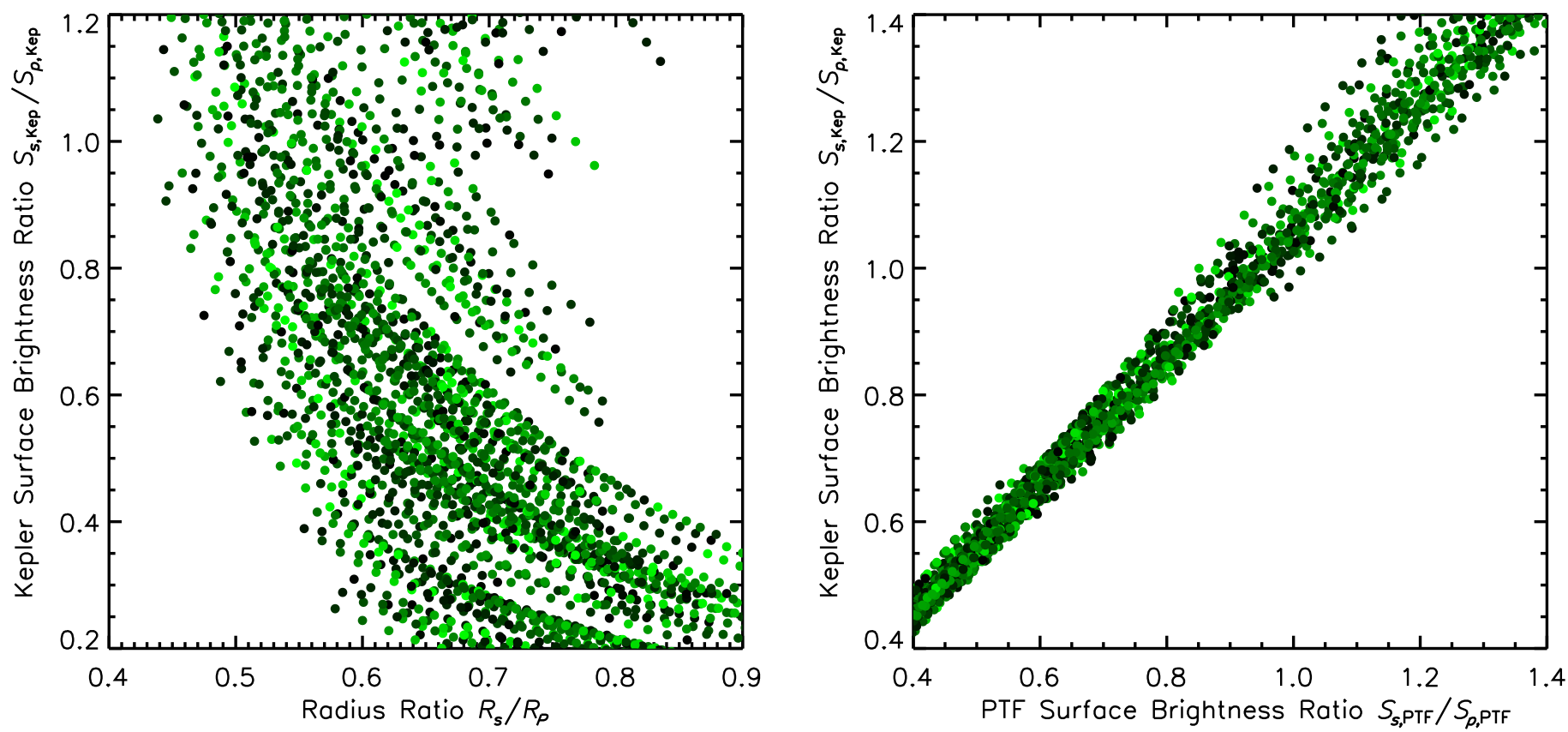

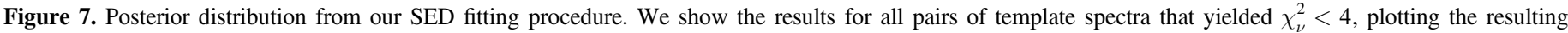

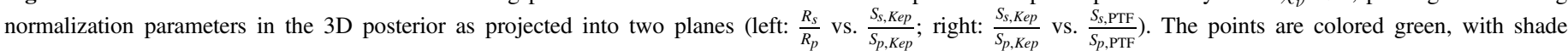

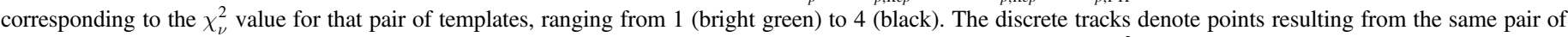

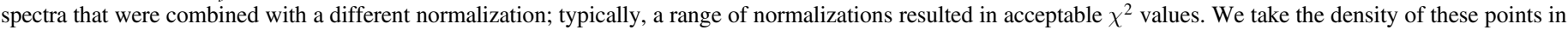

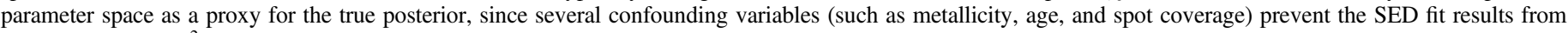
defining a simple $\chi^{2}$ hypersurface.

primary makes it a normal object on the $P_{\text {rot }}-M$ relation for Praesepe (Douglas et al. 2017; Rebull et al. 2017), but it is noticeably faster than the ensemble of field $0.4 M_{\odot}$ stars (e.g., Harrison et al. 2012; McQuillan et al. 2013; Newton et al. 2016), suggesting that it is a suitable representative of a young ZAMS star. There is only a lower limit on the rotational period of the $0.2 M_{\odot}$ secondary; at that limit, it would sit on the slow edge of the Praesepe sequence but would not be unusually slow.

As we show in Figures 11 and 12, the best-fit eccentricity for the system is very small, but it is not zero. This result emerges directly from the $K 2$ light curve. Figure 9 demonstrates that the secondary eclipse is $\sim 10^{-3} P_{\text {orb }}$ ( $\sim 20$ minutes) earlier than the halfway point between primary eclipses. This small eccentricity cannot be detected in the RV curve or PTF light curve alone, and the longitude of periastron is not tightly constrained $\left(\omega=45^{\circ} \pm 25^{\circ}\right)$. An azimuthally asymmetric brightness distribution on one of the stars (due to spots) could cause an apparent shift in the measured eclipse midpoint. The primary star is not tidally locked, though, so an azimuthal asymmetry on that star would cause stochastic variations in eclipse timing, not a constant offset. We cannot rule out this hypothesis for the secondary star, since the lower limit on its rotational period would be consistent with tidal locking.

The surface temperatures of the stars can be inferred in two complementary ways. Our spectroscopic analysis (Section 3.1) yields best-fit temperatures of $T_{\text {eff, } P}=3260 \pm 30 \mathrm{~km} \mathrm{~s}^{-1}$ and $T_{\text {eff }, S}=3120 \pm 50 \mathrm{~K}$, in both cases subject to a $60 \mathrm{~K}(0.5$ subclass) systematic uncertainty from the definition of the underlying grid. Our measurements of the bolometric luminosity and the radius of each star give geometric measurements that are independent of any spectral classification system, albeit with a large uncertainty for the secondary star, yielding $T_{\text {eff }, P}=3290 \pm 70$ and $T_{\text {eff }, S}=2970 \pm 230 \mathrm{~K}$. We therefore find good agreement for the primary to have $T_{\text {eff }} \sim$ $3250-3300 \mathrm{~K}$, while the secondary is most likely $\sim 150 \mathrm{~K}$ cooler than the primary star.

Our measurement of the systemic velocity of PTFEB132.707 $+19.810\left(\gamma=34.00 \pm 0.15 \mathrm{~km} \mathrm{~s}^{-1}\right)$ is consistent with the typical range seen for Praesepe members $\left(v_{\text {rad }} \sim 33-34\right.$ $\mathrm{km} \mathrm{s}^{-1}$; Mermilliod \& Mayor 1999). The proper motion was already known to closely agree; Kraus \& Hillenbrand (2007)

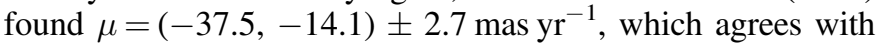
the mean HIPPARCOS value to within $<1 \sigma$. Given the HIPPARCOS distance, we find that the corresponding space velocity for PTFEB132.707+19.810 is $v_{\mathrm{UVW}}=(33.8 \pm$ $1.7,-8.5 \pm 2.2,-2.5 \pm 2.1) \mathrm{km} \mathrm{s}^{-1}$.

Finally, we note that while this paper was under review, PTFEB132.707+19.810 was also reported as an eclipsing binary by Rebull et al. (2017), Douglas et al. (2017), and Gillen et al. (2017). The latter group analyzed eight RV measurements and the $K 2$ light curve to compute system parameters. They reported masses that broadly agree with our results $(\sim 2 \sigma$ smaller, based on mutual uncertainties), as well as a similar primary star radius. However, they reported a significantly smaller secondary star radius $\left(R_{s}=0.226\right.$ versus $R_{s}=0.272 R_{\odot}$, a $3.4 \sigma$ discrepancy); this measurement appears to result from a substantially smaller radius ratio estimate that emerges from their spectroscopic prior. These differences further emphasize the need to understand systematic differences that emerge from different analysis pipelines (Sections 4.2, 4.3, 4.4, and Appendix).

\subsection{The Robustness of Eclipsing Binary Fits}

To test the robustness of our results, we repeated our analysis using only subsets of the data. The RV measurements are always required, since they yield a unique measurement of the 
Table 6

System Parameters for PTFEB132.707+19.810

\begin{tabular}{|c|c|}
\hline \multicolumn{2}{|l|}{ Orbital Parameters } \\
\hline $\begin{array}{l}T_{0}(\mathrm{HJD}) \\
T_{P}(\mathrm{HJD}) \\
P(\text { days }) \\
a(\mathrm{au}) \\
e \\
i(\mathrm{deg}) \\
\omega(\mathrm{deg}) \\
\gamma\left(\mathrm{km} \mathrm{s}^{-1}\right)\end{array}$ & $\begin{aligned} 2457145.0 & \pm 0.4 \\
2457148.9041 & \pm 0.0001 \\
6.015742 & \pm 0.000002 \\
0.05475 & \pm 0.00006 \\
0.0017 & \pm 0.0006 \\
88.87 & \pm 0.05 \\
38 & \pm 27 \\
34.00 & \pm 0.15\end{aligned}$ \\
\hline \multicolumn{2}{|c|}{ Stellar Bulk Parameters } \\
\hline $\begin{array}{l}M_{p}+M_{s}\left(M_{\odot}\right) \\
q=M_{s} / M_{p} \\
M_{p}\left(M_{\odot}\right) \\
M_{s}\left(M_{\odot}\right) \\
R_{p}+R_{s}\left(R_{\odot}\right) \\
R_{s}+R_{p} \\
R_{p}\left(R_{\odot}\right) \\
R_{s}\left(R_{\odot}\right) \\
\end{array}$ & $\begin{aligned} 0.6050 & \pm 0.0020 \\
0.531 & \pm 0.005 \\
0.3953 & \pm 0.0020 \\
0.2098 & \pm 0.0014 \\
0.635 & \pm 0.005 \\
0.75 & \pm 0.05 \\
0.363 & \pm 0.008 \\
0.272 & \pm 0.012\end{aligned}$ \\
\hline \multicolumn{2}{|c|}{ Stellar Atmospheric Parameters } \\
\hline $\begin{array}{l}S_{s, K} / S_{p, K} \\
S_{s, P} / S_{p, P}\end{array}$ & $\begin{array}{c}0.699 \pm 0.006 \\
0.66 \pm 0.04\end{array}$ \\
\hline \multicolumn{2}{|c|}{ Unresolved Stellar Parameters } \\
\hline $\begin{array}{l}F_{\text {bol }}\left(\mathrm{erg} \mathrm{s}^{-1} \mathrm{~cm}^{-2}\right) \\
L_{\text {bol }}\left(L_{\odot}\right)\end{array}$ & $\begin{array}{c}(1.75 \pm 0.06) \times 10^{-11} \\
0.0180 \pm 0.0010\end{array}$ \\
\hline \multicolumn{2}{|c|}{ Primary Star Parameters } \\
\hline $\begin{array}{l}\text { SpT } \\
T_{\text {eff }}(\mathrm{K}) \\
F_{\text {bol }}\left(\mathrm{erg} \mathrm{s}^{-1} \mathrm{~cm}^{-2}\right) \\
L_{\text {bol }}\left(L_{\odot}\right)\end{array}$ & $\begin{aligned} & \text { M3 } 3.5 \pm 0.2 \pm 0.3 \\
& 3260 \pm 30 \pm 60 \\
&(1.32 \pm 0.05) \times 10^{-11}( \pm 2 \%) \\
& 0.0137 \pm 0.0010\end{aligned}$ \\
\hline \multicolumn{2}{|c|}{ Secondary Star Parameters } \\
\hline $\begin{array}{l}\text { SpT } \\
T_{\text {eff }}(\mathrm{K}) \\
F_{\text {bol }}\left(\mathrm{erg} \mathrm{s}^{-1} \mathrm{~cm}^{-2}\right) \\
L_{\text {bol }}\left(L_{\odot}\right)\end{array}$ & $\begin{aligned} & \text { M4.3 } \pm 0.7 \pm 0.3 \\
& 3120 \pm 50 \pm 60 \\
&(0.49 \pm 0.06) \times 10^{-11}( \pm 2 \%) \\
& 0.0050 \pm 0.0015\end{aligned}$ \\
\hline
\end{tabular}

Note. In all cases, we report the median of the marginalized distribution. The values of $T_{0}$ and $\omega$ are individually poorly constrained but are subject to a tight joint constraint that is captured by the time of primary eclipse $T_{P}$. To predict observations from the orbital elements, $\omega$ and $T_{P}$ should be used to compute an appropriate value of $T_{0}$ with sufficient precision. If $\omega, T_{P}, e$, and $P$ are fixed to the values listed in this table, $T_{0}=2457145.0267$. There is a small $(4 \%)$ difference between the system $L_{\mathrm{bol}}$ and the sum of the component $L_{\mathrm{bol}}$ because they are determined from different analysis methods.

component masses. However, the geometric and surface properties are overconstrained by the combination of the $K 2$ light curve, the PTF light curve, and the spectroscopic prior. We therefore can omit subsets of these data while still finding well-bounded posteriors and hence can determine both the importance of each data source and whether all data sources indicate consistent system properties.

We summarize the variation in these properties when fitting different subsets of the full data set in Table 7. We specifically list the eccentricity and inclination of the orbit, the sum and ratio of the stellar radii, the individual stellar radii, and the ratio of the surface brightnesses in the Kepler and PTF bandpasses.

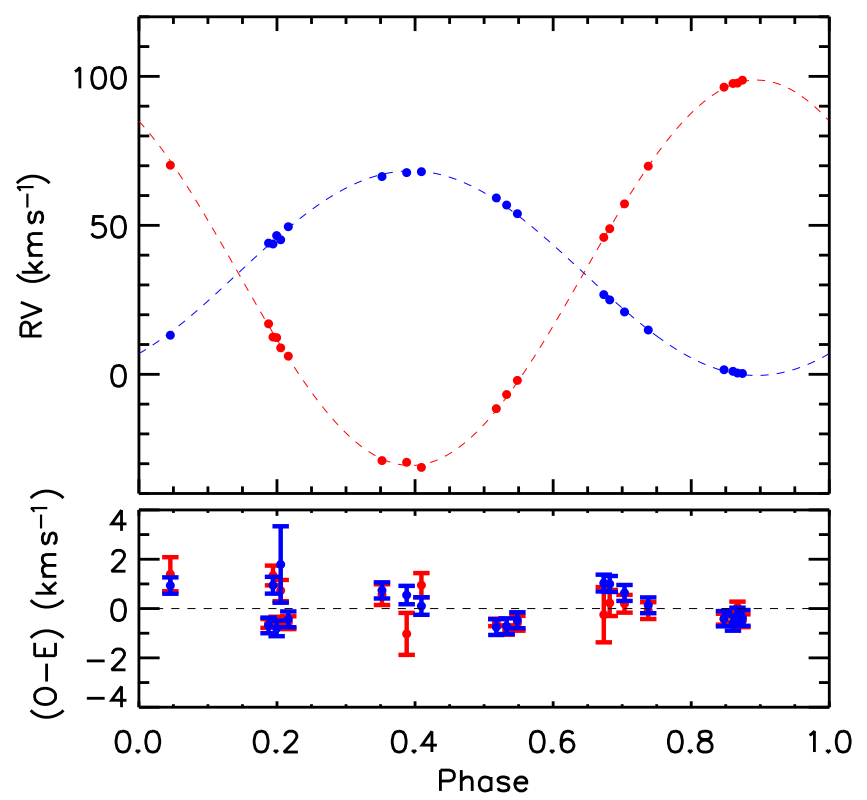

Figure 8. Radial velocities $v_{p}$ (blue) and $v_{s}$ (red) for the primary and secondary stars of PTFEB132.707+19.810, as measured from the Keck/HIRES epochs listed in Table 4. We also show the best-fit model as determined from our fitting procedure (Section 4.1). In the bottom panel, we show the (O-E) residuals with respect to the best-fit model.

We have executed separate MCMC runs by omitting all $K 2$ primary eclipses, all $K 2$ secondary eclipses, all PTF data, or the spectroscopic prior, as well as all combinations thereof that lead to bounded posterior distributions.

The system parameters are surprisingly robust when omitting data sources, changing by $\lesssim 3 \sigma$ and generally with only a modest increase in the uncertainty. There is only a modest impact on the inferred radii. When omitting one data source, the radius fits for the primary star span $0.353-0.377 R_{\odot}$ $( \pm 3.3 \%)$, suggesting that the measurement is robust and all data ultimately point to similar values. The equivalent radius fits for the secondary star span $0.260-0.285 R_{\odot}( \pm 4.5 \%)$, again consistent to within the uncertainties. It is also relevant to consider omission of multiple data sources; while PTFEB132.707 +19.810 is exceedingly well characterized, most systems discovered in $K 2$ or other programs will lack such an abundance of data. When omitting any two data sources, the radius fits for the primary and secondary star span $0.338-0.387 R_{\odot}( \pm 6.8 \%)$ and $0.246-0.308 R_{\odot}( \pm 11 \%)$, respectively. In all cases, the fit parameters inferred from the full data set are centrally located within the range of parameters inferred for the subsets. As we discuss further in Section 4.3, this robustness has strong implications for the impact of spots on our test of the stellar mass-radius relation.

The impact of removing (over)constraints can be seen more clearly in the remaining fit parameters. The sum of the radii, which is strongly constrained by the total duration of the eclipses, remains nearly constant (spanning 0.615-0.647 $R_{\odot}$ ) and well constrained (with error $<3 \%$ ) in all cases. Even fitting only the PTF light curve yields the radius sum with $3 \%$ uncertainty. In contrast, the radius ratio becomes poorly constrained in several subsets and is effectively unconstrained (allowing a ratio above unity at $\lesssim 3 \sigma$ ) when omitting both the prior and another data set. The degeneracy can be avoided for totally eclipsing systems with flat eclipse minima, where the flux ratio securely measures the radius ratio, but few systems meet this geometric requirement. 

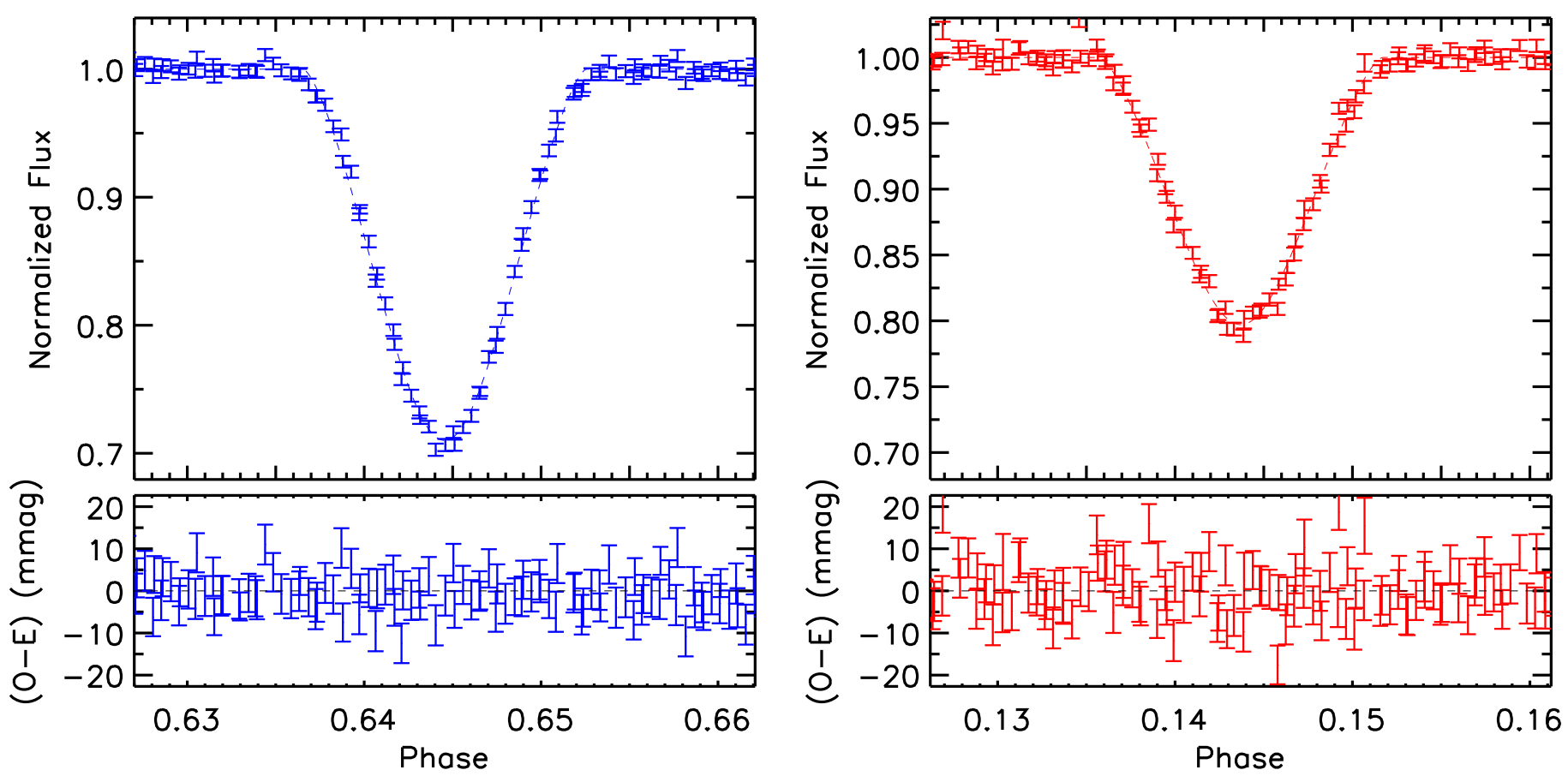

Figure 9. $K 2$ photometry for the primary eclipse (left) and secondary eclipse (right) of PTFEB132.707+19.810, along with the best-fit models (dashed lines) and the (O-E) residuals (bottom panels).
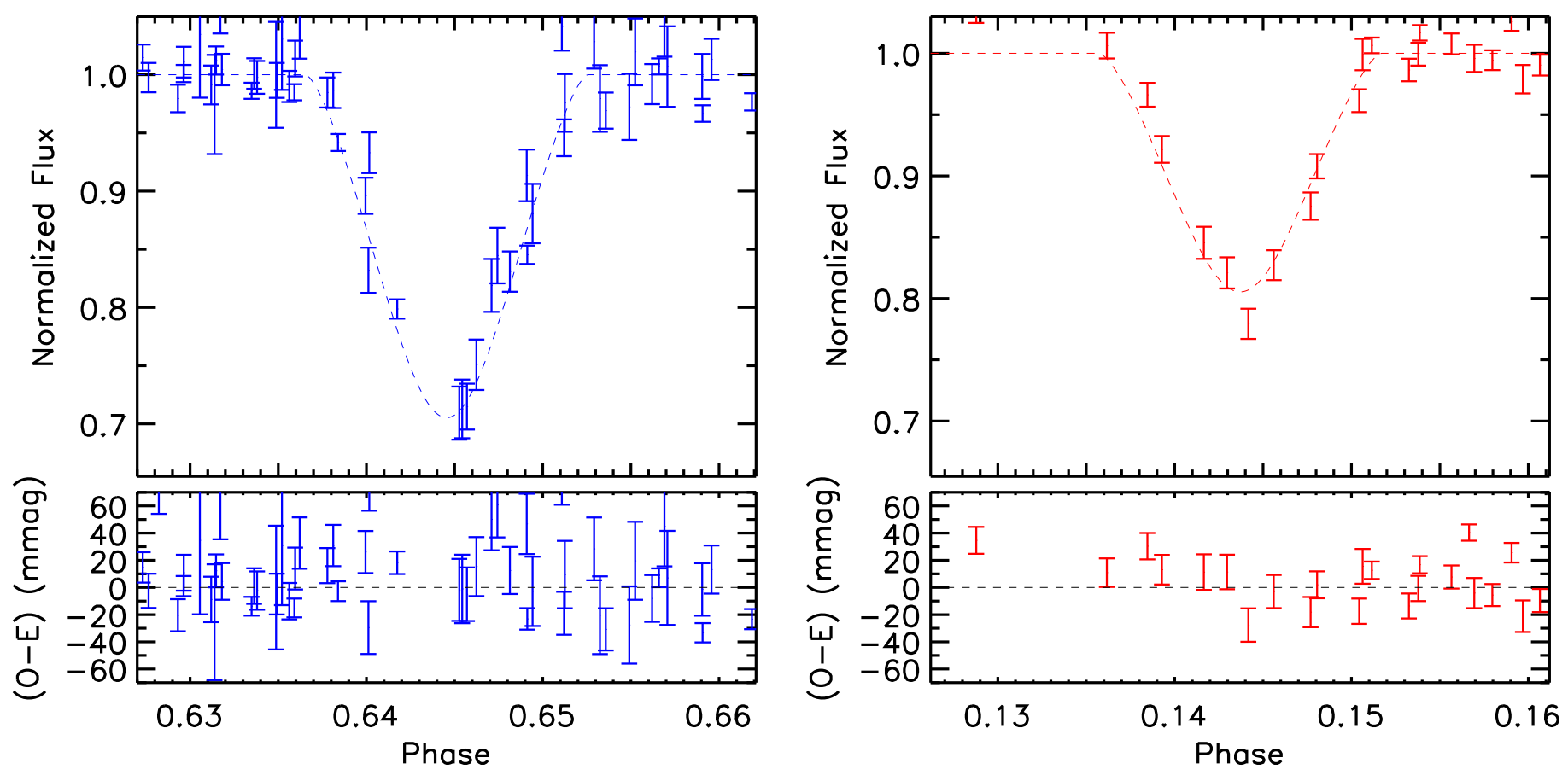

Figure 10. PTF photometry for the primary eclipse (left) and secondary eclipse (right) of PTFEB132.707+19.810, along with the best-fit models (dashed lines) and the $(\mathrm{O}-\mathrm{E})$ residuals (bottom panels).

We therefore suggest that population analyses of known lowmass EBs from the literature (which often are not observed so comprehensively) should compare observed and theoretical radius sums, rather than attempting to consider the two individual stellar radii for each system.

\subsection{The Influence of Spots on Radius Measurements}

M dwarfs commonly host substantial starspots, particularly at young ages (e.g., Cody et al. 2014) or when tidally locked into fast rotation (López-Morales 2007). These starspots complicate the analysis of eclipsing binary light curves. The most obvious impact is that variations in the total spot coverage across both stars will change the out-of-eclipse brightness as a function of rotational phase, requiring rectification in order to properly measure the decrement in brightness specifically due to eclipses. However, the more severe impact for determining detailed stellar properties occurs during eclipse. Changes in relative spot coverage on the occulted area (on the eclipsed 

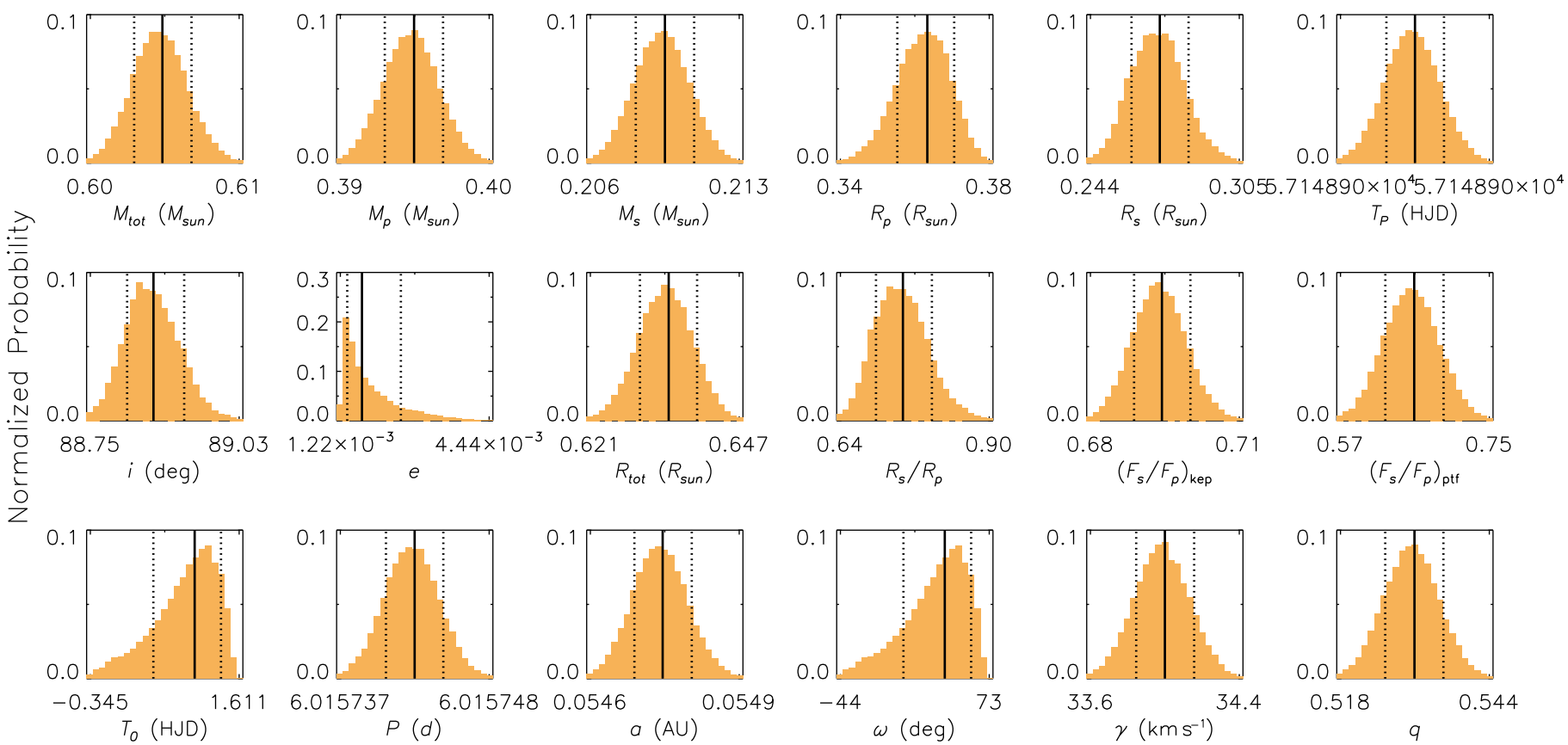

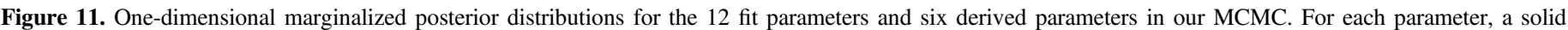
vertical line shows the median, and dotted vertical lines show the central $68 \%$ credible interval.

star) and unocculted areas (on part of the eclipsed star and all of the eclipsing star) will change the overall amplitude of the eclipse, and occultations of individual spots on the background star will introduce high-frequency noise into the eclipse light curve. Most eclipsing binaries have short orbital periods and are tidally locked (Zahn 1977), so the same hemisphere of each star is always visible during eclipse. The effect is therefore identical on all timescales shorter than the spot evolution timescale (i.e., years; Morales et al. 2009; Windmiller et al. 2010), making it difficult to measure the impact or to average out the effect with more data.

PTFEB132.707+19.810 offers a unique opportunity to conduct repeatable tests of the impact of spots on stellar radius determinations. The ratio of the orbital period of PTFEB132.707 +19.810 (6.016 days) and the rotational period of the primary star ( 7.46 days) is very close to $4: 5$, so each primary eclipse occults almost exactly the same range of longitudes (on the primary star) as was occulted 30 days earlier or later. Among the 12 primary eclipses that occurred during the continuous 80 day $K 2$ observation, there are two occultation configurations that recur three times (eclipses $1+6+11$ and $2+7+12$ ) and three that recur twice (eclipses $3+8,4+9$, and $5+10$ ). However, primary eclipse 10 occurred shortly after a flare; we omitted it from our earlier analysis, and while we include it in our tests, we similarly do not factor the results into our conclusions.

To test for potential systematic errors from the unknown spot configuration, we first reran our MCMC 12 times, each time masking all but one primary eclipse to create a subset of the data. We found that the posterior distribution for the system properties did not substantially change, but this result is predictable. As we discussed in Section 4.2, our extensive data set overconstrains the system parameters. If accurate and precise radii can be inferred without any primary eclipses, it naturally follows that any single measurement does not substantially impact the fit, especially since there are only six photometric measurements during each eclipse. However, most systems are unlikely to be characterized this well. We therefore repeated the test with only the $K 2$ data and spectroscopic prior, omitting the PTF data set and using a single $K 2$ primary eclipse at a time.

In Figure 13, we show the resulting posterior distributions for the individual stellar radii using our fit to the $K 2$ light curve and spectroscopic prior, the $K 2$ secondary eclipses and prior, and the intermediately constrained cases with each of the $12 \mathrm{~K} 2$ primary eclipses, the $K 2$ secondary eclipses, and the prior. In each panel, we outline the $68 \%$ credible intervals for the joint posterior on the primary and secondary radii in that case. We find that using fewer data points for the primary eclipse yields larger uncertainties on the stellar radii, as we would expect in a regime where Poisson errors dominate. However, all of the test fits yield similar values to within $1 \sigma$, and most are within $1 \sigma$ of the value when fitting all of them. This result suggests that even though the primary star is heavily spotted (leading to $\pm 5 \%$ total flux variations), changes in the detailed spot configuration do not significantly impact our results. We find an rms scatter of $0.7 \%$ for $R_{p}+R_{s}, 1.8 \%$ for $\frac{R_{s}}{R_{p}}, 0.4 \%$ for $R_{p}$, and $1.7 \%$ for $R_{s}$.

Visual inspection suggests that eclipses of the same hemisphere (denoted by curves with the same color) might more closely resemble each other than the ensemble as a whole, but if so, then only very modestly. If the scatter were purely Poisson but with no systematic effect resulting from sampling five specific spot patterns, then averaging all eclipses of the same hemisphere should reduce the scatter to $\sqrt{\frac{5}{11}}$, or $67 \%$ of these values. We find that the reduction is indeed by approximately this amount; the rms scatter across the five values is reduced to $0.5 \%, 1.0 \%, 0.4 \%$, and $1.0 \%$, respectively, corresponding to $69 \%$ of the original scatter. Our results suggest that any impact from the detailed spot configuration is less than the radius uncertainties resulting from our analysis $(\sim 2 \%)$.

We note that these measurements are unavoidably noisy due to having only 11 eclipses that are distributed between five different 

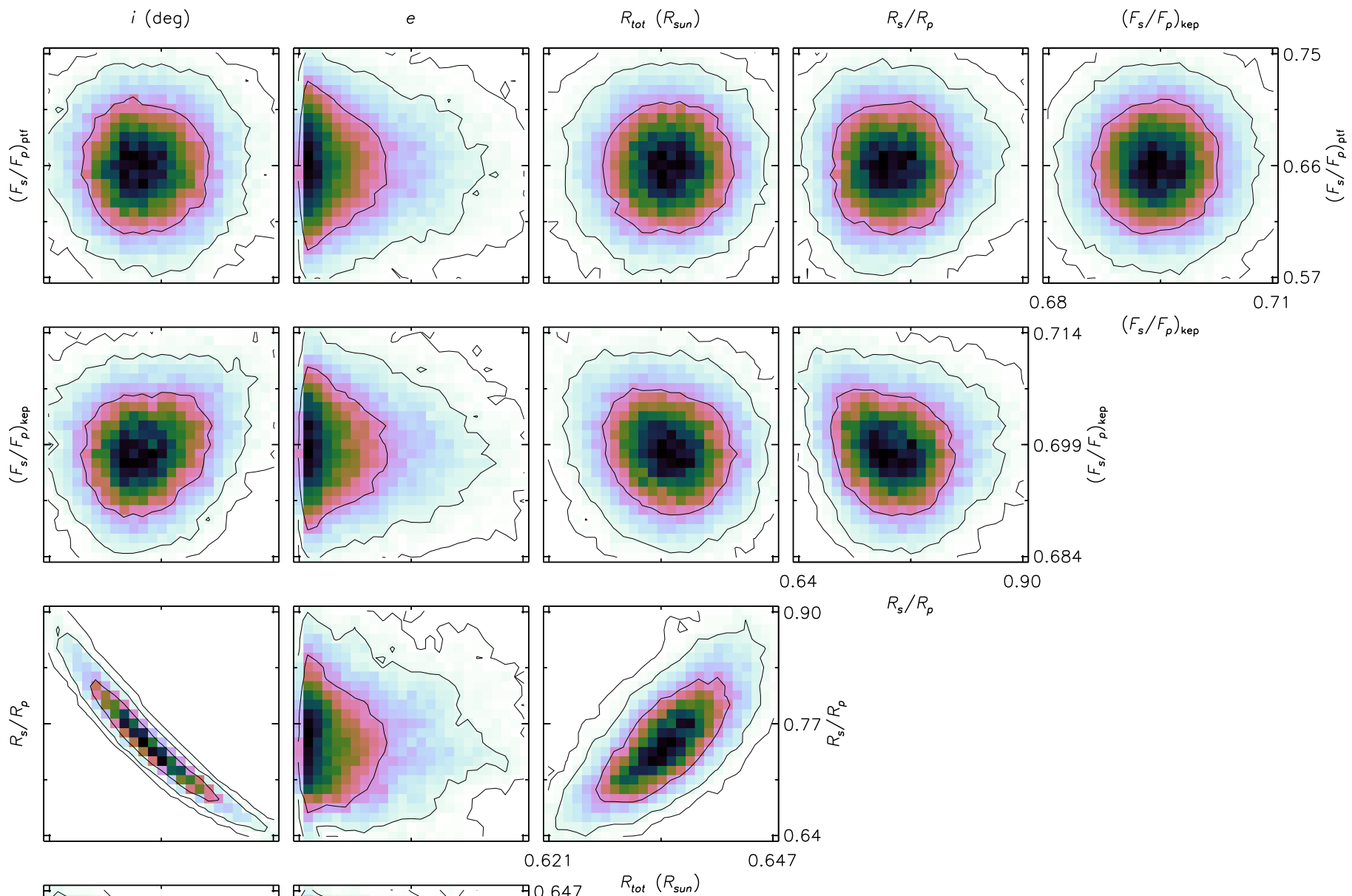

$\left(F_{s} / F_{p}\right)_{\text {kep }}$

$$
R_{s} / R_{p}
$$
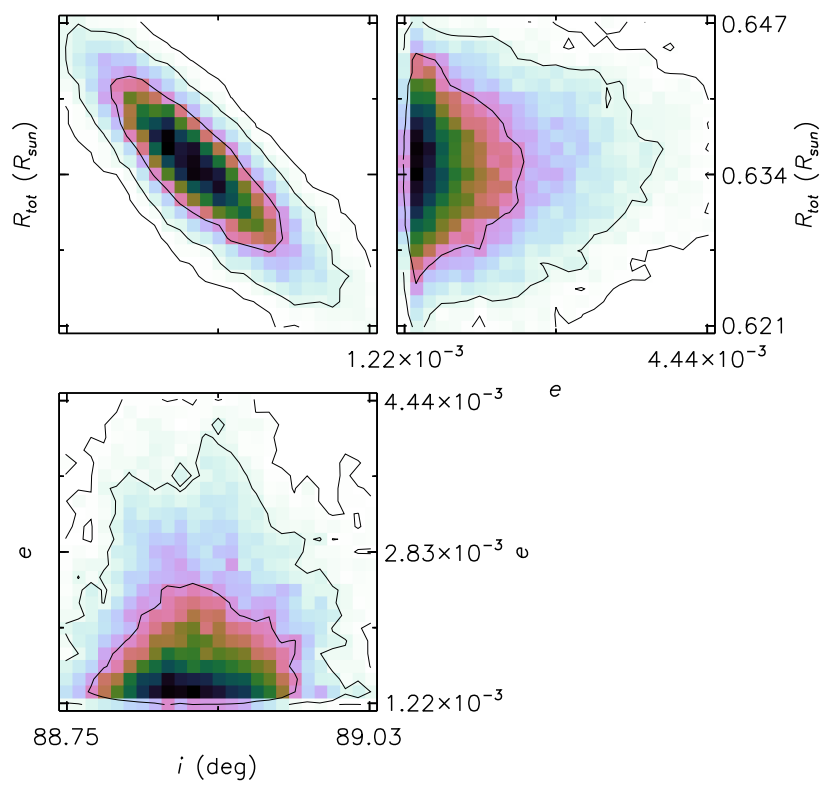

Figure 12. Two-dimensional marginalized posterior distributions for the six fit parameters in our MCMC that are most likely to show astrophysically important covariances. There is a strong covariance between the radius ratio, radius sum, and system inclination (column 1, rows 3 and 4 ; column 3 , row 3 ) and a weaker covariance with the Kepler bandpass surface brightness ratio (column 4, row 2).

spot configuration states, so the exact impact remains uncertain. Given the large uncertainty of the radius ratio and the robustness of the radius sum, unequal-radius systems will see a disproportionately greater impact on the secondary, as we find here. However, we broadly conclude that using optical light curves that sample only a single spot configuration results in a characteristic noise floor of no more than $1 \%-2 \%$, consistent with the results of our earlier simulations (Kraus et al. 2011). Any further reduction of this noise floor would require either observations at longer wavelengths (where spots have lower contrast), sampling multiple spot configurations (by observing systems that are not tidally locked or observing locked systems for longer than the spot evolution timescale), or using outside constraints such as multicolor photometry (such as our use of PTF data). 
Table 7

Variations in System Properties Derived from Data Subsets

\begin{tabular}{|c|c|c|c|c|c|c|c|c|}
\hline Data & $e$ & $\begin{array}{c}i \\
(\operatorname{deg})\end{array}$ & $\begin{array}{l}R_{p}+R_{s} \\
\quad\left(R_{\odot}\right)\end{array}$ & $\frac{R_{s}}{R_{p}}$ & $\begin{array}{c}R_{p} \\
\left(R_{\odot}\right)\end{array}$ & $\begin{array}{c}R_{s} \\
\left(R_{\odot}\right)\end{array}$ & $\frac{\frac{S_{s, \text { Kep }}}{S_{p, \text { Kep }}}}{4}$ & $\frac{S_{s, \text { PTF }}}{S_{p, \text { PTF }}}$ \\
\hline Full Fit & $0.00168 \pm 0.00058$ & $88.871 \pm 0.054$ & $0.6348 \pm 0.0051$ & $0.751 \pm 0.048$ & $0.3626 \pm 0.0080$ & $0.2724 \pm 0.0115$ & $0.699 \pm 0.006$ & $0.659 \pm 0.036$ \\
\hline No Spec Prior & $0.00189 \pm 0.00070$ & $88.820 \pm 0.084$ & $0.6380 \pm 0.0064$ & $0.806 \pm 0.104$ & $0.3533 \pm 0.0168$ & $0.2849 \pm 0.0228$ & $0.700 \pm 0.007$ & $0.676 \pm 0.043$ \\
\hline No PTF & $0.00158 \pm 0.00049$ & $88.874 \pm 0.067$ & $0.6340 \pm 0.0057$ & $0.750 \pm 0.056$ & $0.3626 \pm 0.0091$ & $0.2718 \pm 0.0138$ & $0.699 \pm 0.006$ & $0.622 \pm 0.083$ \\
\hline No $K 2$ Primary & $0.00177 \pm 0.00064$ & $88.863 \pm 0.098$ & $0.6287 \pm 0.0109$ & $0.711 \pm 0.054$ & $0.3676 \pm 0.0078$ & $0.2613 \pm 0.0153$ & $0.747 \pm 0.030$ & $0.676 \pm 0.040$ \\
\hline No $K 2$ Secondary & $0.00178 \pm 0.00063$ & $88.891 \pm 0.087$ & $0.6365 \pm 0.0065$ & $0.688 \pm 0.056$ & $0.3774 \pm 0.0101$ & $0.2601 \pm 0.0147$ & $0.623 \pm 0.061$ & $0.652 \pm 0.036$ \\
\hline No Spec Prior or PTF & $0.00154 \pm 0.00038$ & $88.761 \pm 0.078$ & $0.6398 \pm 0.0057$ & $0.899 \pm 0.186$ & $0.3377 \pm 0.0295$ & $0.3038 \pm 0.0337$ & $0.704 \pm 0.010$ & \\
\hline No Spec Prior or $K 2$ Primary & $0.00166 \pm 0.00068$ & $88.708 \pm 0.126$ & $0.6434 \pm 0.0133$ & $0.909 \pm 0.329$ & $0.3386 \pm 0.0460$ & $0.3076 \pm 0.0579$ & $0.773 \pm 0.063$ & $0.690 \pm 0.046$ \\
\hline No Spec Prior or $K 2$ Secondary & $0.00178 \pm 0.00066$ & $88.845 \pm 0.122$ & $0.6392 \pm 0.0080$ & $0.696 \pm 0.093$ & $0.3775 \pm 0.0167$ & $0.2625 \pm 0.0235$ & $0.551 \pm 0.088$ & $0.681 \pm 0.044$ \\
\hline No PTF or $K 2$ Primary & $0.00108 \pm 0.00094$ & $88.992 \pm 0.274$ & $0.6152 \pm 0.0165$ & $0.671 \pm 0.070$ & $0.3667 \pm 0.0175$ & $0.2464 \pm 0.0176$ & $0.720 \pm 0.182$ & $0.658 \pm 0.207$ \\
\hline No PTF or $K 2$ Secondary & $0.00088 \pm 0.00081$ & $88.850 \pm 0.138$ & $0.6385 \pm 0.0083$ & $0.680 \pm 0.062$ & $0.3790 \pm 0.0116$ & $0.2582 \pm 0.0165$ & $0.523 \pm 0.200$ & $0.458 \pm 0.210$ \\
\hline No $K 2$ Primary or $K 2$ Secondary & $0.00179 \pm 0.00086$ & $88.843 \pm 0.152$ & $0.6472 \pm 0.0232$ & $0.673 \pm 0.079$ & $0.3867 \pm 0.0175$ & $0.2606 \pm 0.0236$ & $0.744 \pm 0.104$ & $0.679 \pm 0.045$ \\
\hline Only PTF & $0.00172 \pm 0.00099$ & $88.638 \pm 0.139$ & $0.6619 \pm 0.0230$ & $0.974 \pm 0.338$ & $0.3384 \pm 0.0521$ & $0.3302 \pm 0.0571$ & $\ldots$ & $0.698 \pm 0.048$ \\
\hline Only $K 2$ Primary & $0.00090 \pm 0.00086$ & $88.850 \pm 0.150$ & $0.6381 \pm 0.0089$ & $0.739 \pm 0.155$ & $0.3690 \pm 0.0287$ & $0.2726 \pm 0.0347$ & $0.641 \pm 0.315$ & $\cdots$ \\
\hline Only $K 2$ Secondary & $0.00142 \pm 0.00116$ & $88.809 \pm 0.252$ & $0.6324 \pm 0.0203$ & $0.797 \pm 0.251$ & $0.3489 \pm 0.0359$ & $0.2811 \pm 0.0528$ & $0.735 \pm 0.135$ & \\
\hline
\end{tabular}



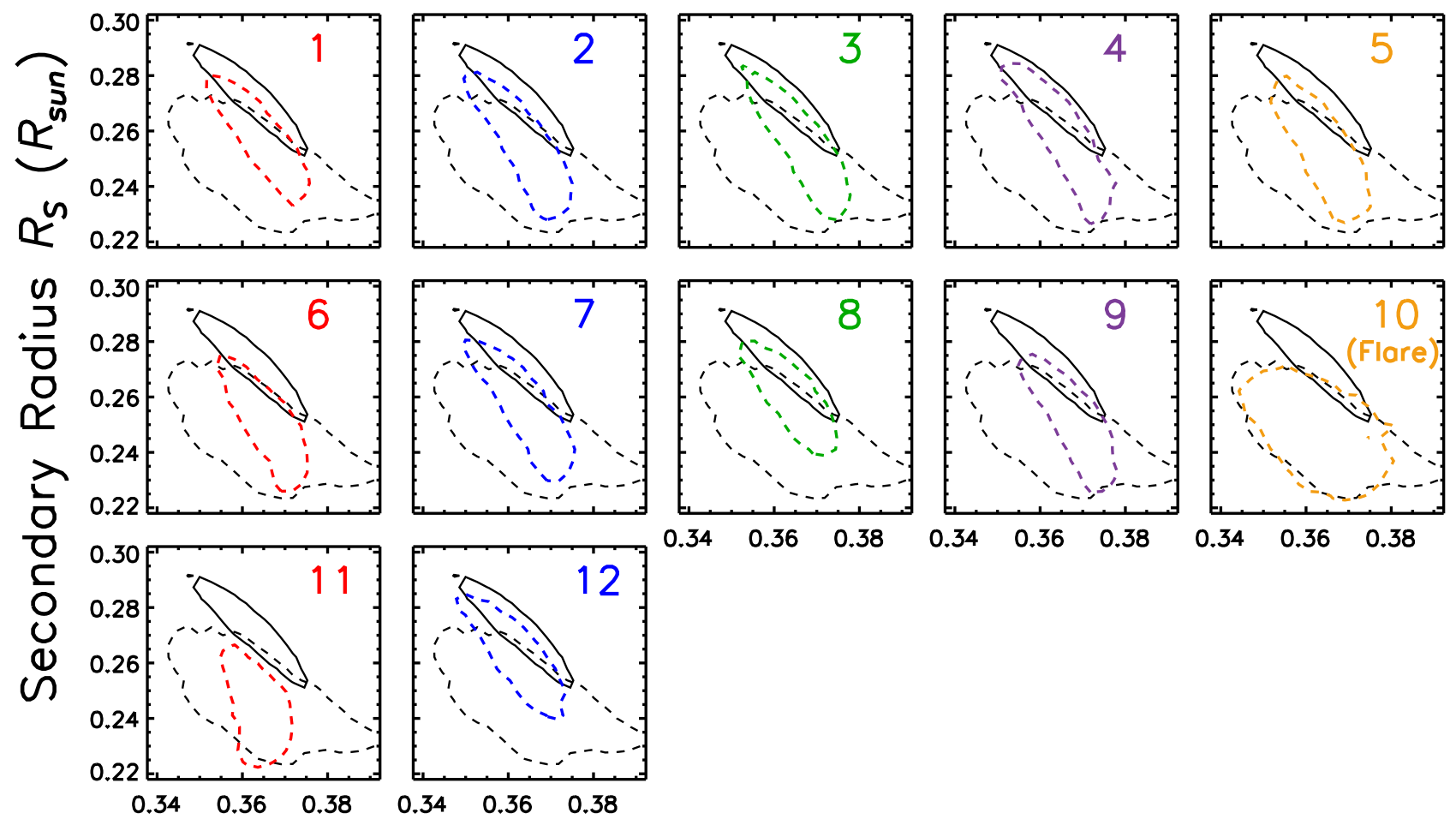

\section{Primary Radius $R_{p}\left(R_{\text {sun }}\right)$}

Figure 13. Joint credible intervals (drawn at 68.7\%) on the stellar radii of PTFEB132.707+19.810 A+B, as derived from subsets of the data. We show the intervals when fitting the $K 2$ light curve, spectroscopic prior, and RVs (solid black contours); only the $K 2$ secondary eclipses, spectroscopic prior, and RVs (dashed black contours); and the credible intervals using only one $K 2$ primary eclipse, $K 2$ secondary eclipses, spectroscopic prior, and RVs (dashed colored contours). Each panel shows the result for using only one primary eclipse, and the single-eclipse fits are color-coded to denote eclipses that occulted the same range of longitudes on the primary star: 1/6/11 (red), 2/7/12 (blue), 3/8 (green), 4/9 (purple), and 5/10 (orange). Eclipse 10 occurred just after a flare, so we do not include it in any analysis.

\subsection{Systematic Uncertainties in Radii from Limb-darkening Models}

The morphology of an eclipse light curve and the inferred stellar radii also depend on the stellar limb darkening, since a limb-darkened star would be visually similar to a uniformly illuminated disk of smaller radius. Nearly the same eclipse can result from a degenerate combination of possible limbdarkening laws, stellar radii, and orbital inclinations, so assumptions about the limb-darkening law will be reflected directly in the resulting radius measurements. Due to the paucity of spatially resolved images of stars, limb-darkening laws are typically derived from radiative transfer through model atmospheres, but different treatments yield prescriptions that differ by $\sim 0.1$ even just in the linear term (e.g., Claret \& Bloemen 2011 versus Sing 2010). Some light-curve analyses attempt to bypass the theoretical uncertainties by directly fitting for the limb-darkening coefficients, but these empirical measurements do not yield consistent results between different stars and can sometimes even imply naively unphysical results such as limb brightening. The empirical analyses might themselves be limited by spatial inhomogeneities in the surface brightness of the stars (such as from spots and plages) that can change the de facto limb darkening of the star, especially in the case of spatially coherent spot patterns such as polar spots (Morales et al. 2009).

As with our treatment of spots, we conservatively adopt a uniform theoretical treatment of limb darkening but then quantify the resulting systematic uncertainty in our radius measurements that could result from potential variations in the limb-darkening law. To that end, we have repeated our analysis of the data set using three other assumptions for limb darkening. To establish a baseline worst-case scenario, we first removed all limb-darkening effects and treated the stars as uniformly illuminated disks. We then repeated our analysis twice more with quadratic limb darkening in place but with the linear coefficients increased or decreased by 0.1 , which establishes a more relevant range of likely radius uncertainties.

In Figure 14, we show the resulting posterior distributions for the individual stellar radii using our default limb-darkening values and the three modified sets of assumptions. In each case, we outline the $68 \%$ credible interval for the joint posterior on the primary and secondary radii. As expected, we find that the radius depends on the assumed limb-darkening law; removing limb darkening entirely results in stars that are $\sim 6 \%$ smaller, while the more realistic variations in the linear term result in $\pm 1 \%-2 \%$ changes in the inferred radius sum and individual radii. We find that for this system, most of the impact from small changes in limb darkening is reflected in the radius of the secondary star. In cases where limb darkening varies from star to star (such as from spots), the individual radii and radius ratio vary more substantially. However, the broad conclusion is that uncertainties from limb darkening are of order $\sigma_{R} \sim 1 \%-2 \%$; hence, they are no larger than the other systematic effects we discuss.

\section{Confronting Stellar Evolutionary Models}

Evolutionary models of M dwarfs are fundamentally a set of relations between independent variables that are fixed for a 


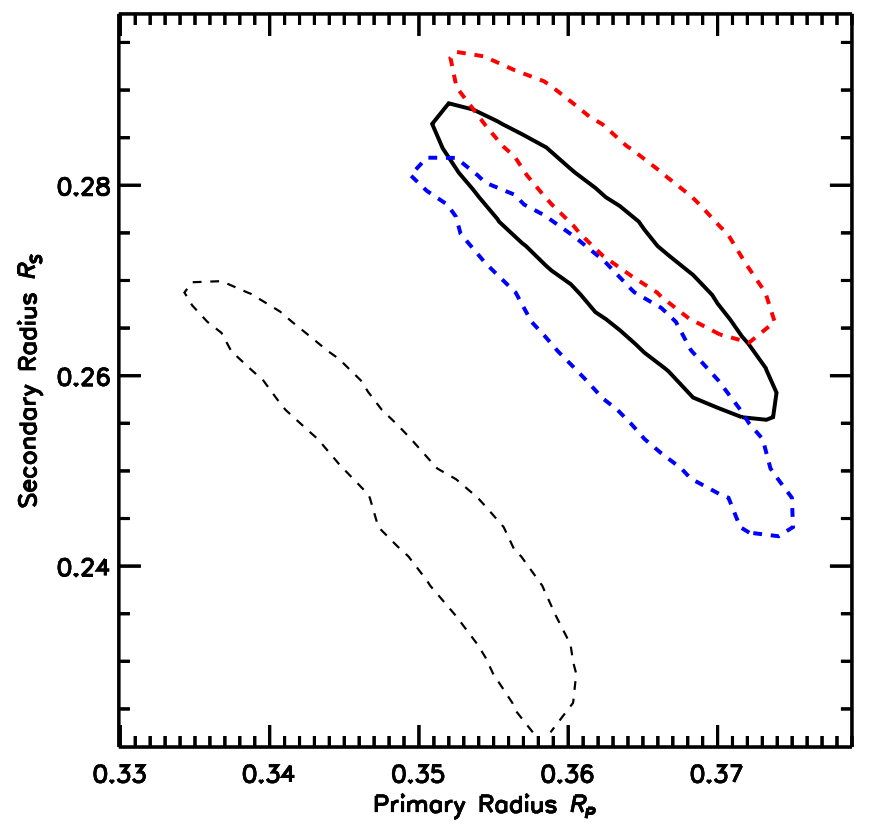

Figure 14. Joint credible intervals (drawn at 68.7\%) on the stellar radii of PTFEB132.707+19.810 A+B, as derived using different assumptions about stellar limb darkening. The solid black contour shows the results for our adopted limbdarkening parameters, while the red and blue dashed contours represent a change in the linear coefficient of $\Delta u_{1}=0.1$ upward or downward, as is typically seen between different theoretical treatments of limb darkening (e.g., Claret \& Bloemen 2011 vs. Sing 2010). The black dashed contour shows a fit with no limb darkening, hence treating the stars as uniformly illuminated disks. The red and blue contours show that for typical variations in adopted limb-darkening parameters, the resulting systematic uncertainty in the sum of the radii and individual radii is $\sim 1 \%-2 \%$. For this specific system, most of the impact from small changes in limb darkening is reflected in the radius of the secondary. Even in the worst-case scenario of using no limb darkening, the stars are only $6 \%$ smaller, with similar impact on both stars. We therefore conclude that differences in the detailed treatment of limb darkening cannot explain the observed scatter in the field mass-radius relation $( \pm 5 \%)$ or the radius discrepancy we see for the secondary star $(20 \%)$.

given star (mass and metallicity), prescriptions for linked features that evolve over time (rotation, magnetic field strength, and spot fraction), and predicted dependent variables (radius, $T_{\text {eff, and luminosity). These models are calibrated using }}$ observations that probe different combinations of the independent, prescriptive, and dependent variables. Examples include panchromatic spectroscopy to calibrate the luminosity- $T_{\text {eff }}$ relation (e.g., Mann et al. 2015), long-baseline interferometry to calibrate the luminosity- $T_{\text {eff }}$-radius relation (e.g., Boyajian et al. 2012), and monitoring of visual binaries to calibrate the luminosity- $T_{\text {eff }}$-mass relation (Delfosse et al. 2000; Benedict et al. 2016). All of these tests can be conducted in stellar populations to further probe metallicity and age. Eclipsing binaries pose a unique test of evolutionary models, though, offering the only direct relation between mass and radius while also being amenable to the measurement of luminosity and $T_{\text {eff }}$.

In Figure 15 (left), we plot the locations of the binary components in the HR diagram, along with the coeval isochrones of the BHAC15 (Baraffe et al. 2015) and Dartmouth Stellar Evolutionary Program (DESP; Dotter et al. 2008) stellar evolutionary models; for the latter, we show tracks at solar metallicity and the metallicity of Praesepe $([\mathrm{Fe} / \mathrm{H}]=0.14)$. We also denote the model-predicted luminosities and temperatures for each component, given our dynamical mass measurements. We find that both stars are predicted to be hotter than the measured temperatures, and the primary is also predicted to be more luminous than its measured luminosity.
In Figure 15 (right), we plot the locations of the binary components in the mass-radius diagram, also in comparison to the BHAC15 and DSEP models. The radius of the primary star appears to be predicted well by both sets of theoretical models, with a discrepancy within the observational uncertainty. However, both sets of theoretical models predict a significantly smaller radius for the secondary $\operatorname{star}\left(R \sim 0.22 R_{\odot}\right)$ than we empirically measure $\left(R=0.272 \pm 0.012 R_{\odot}\right)$, disagreeing by $\sim 0.05 R_{\odot}$, or $\sim 20 \%$.

The unexpected cool temperature of the primary star appears to be robust, since it dominates the observed flux. Past observations of the (unresolved) system have found a spectral type of M3.4 from the broadband SED (Kraus \& Hillenbrand 2007) and M5 from the optical spectrum (West et al. 2011). In comparison, the model-predicted temperature of $T_{\text {eff }} \sim 3500 \mathrm{~K}$ corresponds to a spectral type of M1.5 (Casagrande et al. 2008) to M2 (Rajpurohit et al. 2013), depending on the temperature scale used. The observed presence of VO bands at 7600-7800 $\AA$ makes the distinction unambiguous, since they are not present for $\leqslant \mathrm{M} 2$ stars. Furthermore, the CMD for the rest of Praesepe shows a similar tendency for members to be systematically redder/cooler than models, as demonstrated in Figure 3 of Mann et al. (2017).

The HR diagram positions are inferred via a method that is almost entirely independent of the radius measurements, so it further bolsters the reliability of our analysis that the discrepancies for the well-characterized primary star are of appropriate sign and magnitude to preserve the Stefan-Boltzmann law $\left(L=4 \pi R^{2} \sigma T^{4}\right)$. The radius is consistent with theoretical predictions, and the discrepancy in predicted temperature $(250 \pm 30 \mathrm{~K}$, or $\sim 7 \%)$ is almost exactly offset by the discrepancy in predicted luminosity $\left(0.004 \pm 0.001 L_{\odot}\right.$, or $\left.\sim 28 \%\right)$. The combined properties therefore indicate a mutually consistent discrepancy with respect to models. We cannot conduct this consistency check for the secondary star because the fractional luminosity uncertainty $(\sim 30 \%)$ is too large for a meaningful comparison.

The origin of these discrepancies remains unclear and will be difficult to assess with only a single well-characterized system. A number of hypotheses can be deemed unlikely. Models computed at $[\mathrm{Fe} / \mathrm{H}]=0$ and $[\mathrm{Fe} / \mathrm{H}]=0.14$ (appropriate for Praesepe) give virtually identical predictions for stellar parameters, so metallicity mismatch does not seem to be significant. Stassun et al. (2014) found among young eclipsing systems that radii were larger than model predictions in triple systems, which might suggest an origin in dynamically driven binary interactions. We do not have adaptive optics imaging for this system, but the absence of a third broadening function peak suggests that any tertiary companion would need to be substantially fainter ( 22 mag at $8000 \AA$ ). Finally, Praesepe is old enough that even the secondary star should have reached the ZAMS much earlier in its lifetime (Dotter et al. 2008; Baraffe et al. 2015), so it is also unlikely that the secondary is still undergoing pre-main-sequence contraction.

However, while this system is likely on the ZAMS, it is still substantially younger than the field population. Old field stars typically show smaller discrepancies or even agree with models (Torres et al. 2010; Kraus et al. 2011; Feiden \& Chaboyer 2014b). Young stars are known to be more active than their older counterparts (e.g., West et al. 2008), suggesting a possible role for magnetic fields (Chabrier et al. 2007; Feiden \& Chaboyer 2014a; Mullan \& MacDonald 2001; MacDonald \& Mullan 2014) or starspots (Somers \& Pinsonneault 2015) in changing the interior structures of stars or the course of stellar evolution. Magnetism only seems to change the stellar parameters of fully convective main-sequence stars if interior magnetic field strengths are far 

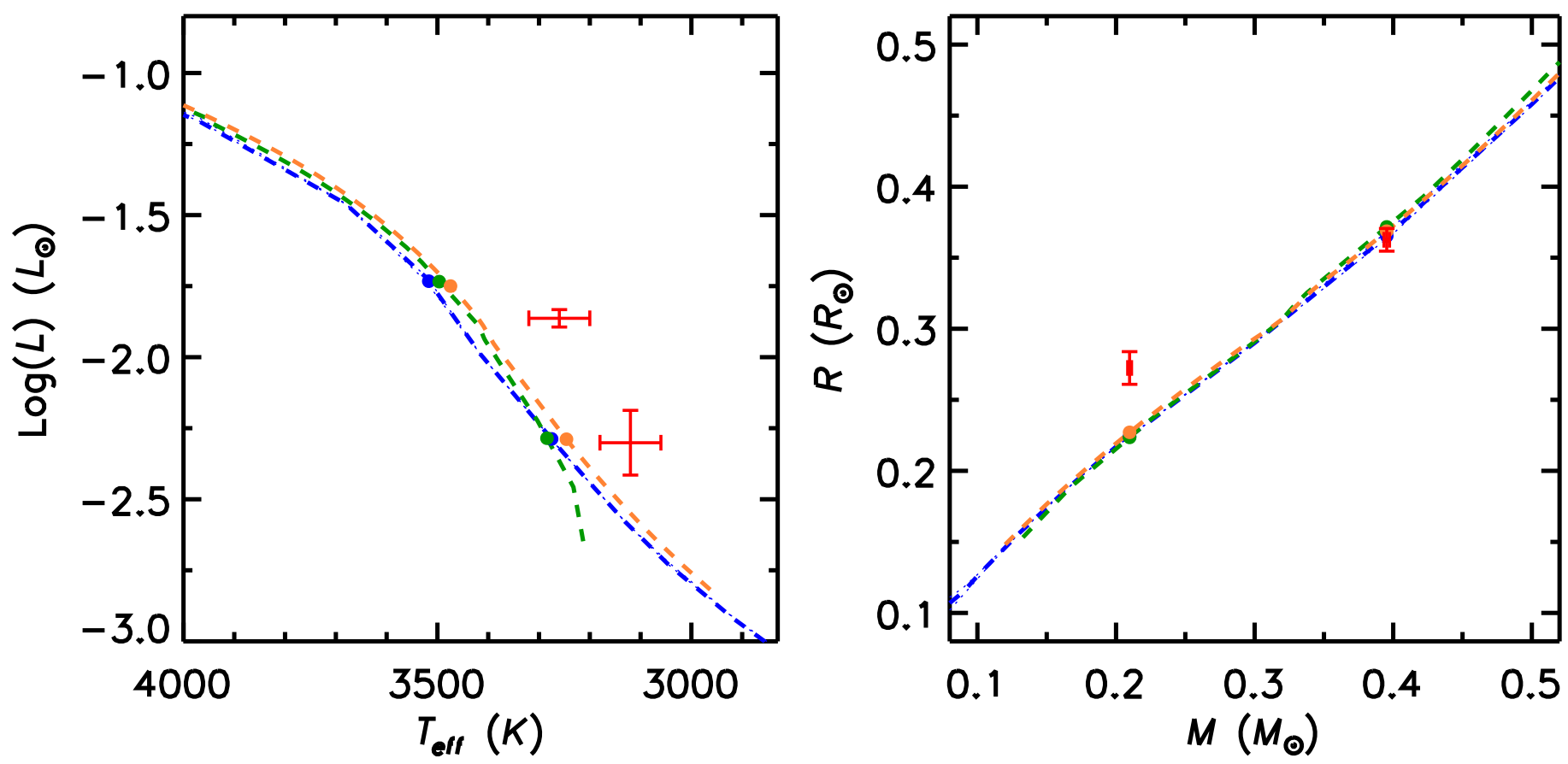

Figure 15. Left: the $L-T_{\text {eff }}$ HR diagram showing our spectroscopic measurements for the components of PTFEB132.707+19.810 (red) and the theoretical isochrones at $\tau=600 \mathrm{Myr}$ for BHAC15 (blue), DSEP with $[\mathrm{Fe} / \mathrm{H}]=0$ (green), and DSEP with $[\mathrm{Fe} / \mathrm{H}]=0.14$ (orange). The interpolated model positions for our measured masses of the two stars are shown with filled circles. The models predict substantially higher temperatures $\left(\Delta T_{\text {eff }} \sim 150-250 \mathrm{~K}\right)$ than those that result from our spectroscopic analysis or the geometric $T_{\text {eff }}$ corresponding to our radius measurements. Right: mass-radius diagram showing the components of PTFEB132.707 +19.810 and the predictions of the BHAC15 and DSEP models at $\tau=600 \mathrm{Myr}$. For both model sets, the primary agrees well with the mass-radius relations, but the secondary star's radius is significantly underpredicted $(\Delta R \sim 20 \%)$ by theory. For both the HR and mass-radius diagram, metallicity does not change the model tracks by a sufficient amount to explain the observed discrepancies. The BHAC15 isochrones for $\tau=500 \mathrm{Myr}$ and $\tau=1 \mathrm{Gyr}$ fall underneath the $\tau=625 \mathrm{Myr}$ isochrone in these plots, so uncertainty in the age of Praesepe (e.g., Brandt \& Huang 2015) also cannot explain the discrepancies.

higher than expected $(B>1 \mathrm{MG}$; Feiden \& Chaboyer 2014a, 2014b), though some models suggest a lower threshold (MacDonald \& Mullan 2014). The corresponding surface magnetic field $(B \sim 10 \mathrm{kG})$ may be possible in the cores of spots but exceeds the thermal equipartition value and is unlikely to represent a global average value (e.g., Shulyak et al. 2011, 2014). The impact of starspots on stellar evolution in mid-M dwarfs should be explored further in theoretical models, though existing treatments suggest that they do not delay pre-main-sequence evolution to the age of Praesepe or maintain steady-state radius excesses as large as 20\% (e.g., Spruit \& Wiess 1986; Jackson \& Jeffries 2014).

Our measurements indicate that the stars in PTFEB132.707 +19.810 are not unusually active or rapidly rotating compared to other Praesepe members (e.g., Douglas et al. 2014). Given a system flux ratio of $\sim 3: 1$ at $\lambda=6500 \AA$, the individual $\mathrm{H} \alpha$ equivalent widths for each star are $\mathrm{EW}[\mathrm{H} \alpha]_{P} \sim-4$ and $\mathrm{EW}[\mathrm{H} \alpha]_{S} \sim-2 \AA$; both measurements fall on the massactivity sequence of Praesepe members (from Figure 5 of Douglas et al. 2014), and indeed the secondary falls at the less active edge of the $\mathrm{H} \alpha$ distribution for its mass. Similarly, the rotational period of the primary $\left(P_{P}=7.46\right.$ days $)$ falls on the cluster mass-rotation sequence, while the rotation period of the secondary $\left(P_{S} \gtrsim 6\right.$ days) is at or beyond the slow edge of the distribution for its mass. The empirical relations of Stassun et al. (2014) would still predict from the $\mathrm{H} \alpha$ emission of the secondary star that it should have a radius which is $10 \%$ larger and a temperature which is 5\% smaller than for old field stars, which could partially explain our results. However, we do not find any evidence that the disagreement. We therefore do not find any evidence that the disagreement with models should be limited to this system. Given that the entire
Praesepe HR diagram sequence is found to be cooler than models predict, it is likely that the tension with models is a common feature of all low-mass stars at this age.

\section{Summary and Conclusions}

We have discovered and characterized PTFEB132.707 +19.810 , a $P_{\text {orb }}=6.0$ day eclipsing binary system in the Praesepe open cluster. We find that the system comprises two late-type stars $\left(S p T_{P}=M 3.5 \pm 0.2 ; S p T_{S}=M 4.3 \pm 0.7\right)$ with precisely measured masses $\left(M_{p}=0.3953 \pm 0.0020 M_{\odot} ; M_{s}=\right.$ $\left.0.2098 \pm 0.0014 M_{\odot}\right)$ and radii $\left(R_{p}=0.363 \pm 0.008 R_{\odot}\right.$; $\left.R_{s}=0.272 \pm 0.012 R_{\odot}\right)$. Based on tests using subsets of our data, we find that the results are consistent to within $2 \%-3 \%$ even when omitting some data sets. We also find that at least the primary star is not tidally locked to the orbital period, and we take advantage of the 4:5 ratio of $P_{\text {orb }}$ to $P_{\text {rot }, P}$ to conduct a natural experiment in the variance and repeatability of radius measurements due to different spot configurations on the occulted star. We demonstrate that the full analysis is resilient to changes in spot variation (changing the inferred radii by $\lesssim 1 \%$ ); for data volumes more typical of EB studies, the scatter in inferred radii is still no more than 1\%-2\%, implying a noise floor in the characterization of most eclipsing systems. We also test different assumptions for limb darkening and show that for a plausible range of parameters, the stellar radii only change by $1 \%-2 \%$.

Given the masses, neither star meets the predictions of stellar evolutionary models of the appropriate age and metallicity. The primary star has the expected radius, but it is cooler and less luminous than models would predict; the secondary star has the expected luminosity, but it is cooler and substantially larger (by $20 \%$ ) than models would predict. These results broadly match the 
known discrepancy between theoretical models and Praesepe's empirical cluster sequence in the HR diagram, and the mass and radius measurements now reveal the magnitude of the discrepancies in each quantity. These discrepancies are larger than those for old field stars that also are still on the ZAMS, suggesting that age-dependent effects beyond pre-main-sequence contraction and nuclear burning (such as rotational spindown and the decay of stellar activity) must be incorporated in stellar evolutionary models. Moreover, the primary and secondary stars span the fully convective boundary, so the different forms of discrepancy might indicate that the existence of a convective-radiative boundary influences the extra physics, as would be expected if tied to generation and field strength of the stellar magnetic fields.

Finally, we validated our pipeline by analyzing extant data for the well-studied system GU Boo, showing that our very different analysis methods match previous radius measurements to within $2 \%-3 \%$. A similar result was found for GU Boo and yet another pipeline by Windmiller et al. (2010). Given the wide variety of analysis methods used in literature studies of low-mass eclipsing systems, we suggest that interpretation of the stellar mass-radius relation should include a systematic term of $2 \%-3 \%$, unless all analyses were conducted with similar observations and analysis pipelines.

We thank Phil Muirhead and Eunkyu Han for interesting discussions on low-mass eclipsing binaries and comparing results on our respective pipelines, Trent Dupuy for helpful discussions on the fitting of orbits, and Lynne Hillenbrand for contributing to the Keck/HIRES data acquisition. We also thank the referee for providing a helpful and thoughtful critique of our paper that led to a number of valuable additions to the manuscript. AWM was supported through Hubble Fellowship grant 51364 awarded by STScI, which is operated by AURA for NASA, under contract NAS 5-26555. GT acknowledges partial support for this work from NSF grant AST-1509375. MAA acknowledges support provided by the NSF through grants AST-1255419 and AST-1517367.

This paper includes data collected by the $K 2$ mission. Funding for the $K 2$ mission is provided by the NASA Science Mission directorate. The $K 2$ data presented in this paper were obtained from the Mikulski Archive for Space Telescopes. Funding for the Sloan Digital Sky Survey IV has been provided by the Alfred P. Sloan Foundation, the U.S. Department of Energy Office of Science, and the Participating Institutions. SDSS-IV acknowledges support and resources from the Center for High-Performance Computing at the University of Utah. The SDSS web site is http://www.sdss.org. SDSS-IV is managed by the Astrophysical Research Consortium for the Participating Institutions of the SDSS Collaboration.

This research has also made use of the Keck Observatory Archive (KOA), which is operated by the W.M. Keck Observatory and the NASA Exoplanet Science Institute (NExScI), under contract with the National Aeronautics and Space Administration. Some of the data presented herein were obtained at the W.M. Keck Observatory, which is operated as a scientific partnership among the California Institute of Technology, the University of California, and the National Aeronautics and Space Administration. The observatory was made possible by the generous financial support of the W.M. Keck Foundation.

The authors wish to recognize and acknowledge the very significant cultural role and reverence that the summit of Maunakea has always had within the indigenous Hawaiian community. We are most fortunate to have the opportunity to conduct observations from this mountain.
Table 8

System Parameters for GU Boo Based on Data from LMR05

\begin{tabular}{lc}
\hline \hline Orbital Parameters & \\
\hline$T_{0}(\mathrm{HJD})$ & $2452723.98150 \pm 0.00006$ \\
$T_{P}(\mathrm{HJD})$ & $2452723.98150 \pm 0.00006$ \\
$P($ days $)$ & $0.4887279 \pm 0.0000008$ \\
$a(\mathrm{au})$ & $0.012934 \pm 0.000009$ \\
$e$ & 0 \\
$i(\mathrm{deg})$ & $87.464 \pm 0.075$ \\
$\omega(\mathrm{deg})$ & 270 \\
$\gamma\left(\mathrm{km} \mathrm{s}{ }^{-1}\right)$ & $-24.25 \pm 0.11$ \\
\hline Stellar Bulk Parameters & \\
\hline$M_{p}+M_{s}\left(M_{\odot}\right)$ & $1.2084 \pm 0.0024$ \\
$q=M_{s} / M_{p}$ & $0.981 \pm 0.002$ \\
$M_{p}\left(M_{\odot}\right)$ & $0.6099 \pm 0.0017$ \\
$M_{s}\left(M_{\odot}\right)$ & $0.5985 \pm 0.0010$ \\
$R_{p}+R_{s}\left(R_{\odot}\right)$ & $1.2354 \pm 0.0032$ \\
$R_{s}+R_{p}$ & $1.051 \pm 0.021$ \\
$R_{p}\left(R_{\odot}\right)$ & $0.6023 \pm 0.0068$ \\
$R_{s}\left(R_{\odot}\right)$ & $0.6332 \pm 0.0060$ \\
\hline Stellar Atmospheric Parameters & \\
\hline$S_{s, \text { Ic }} / S_{p, \text { Ic }}$ & \\
$S_{s, \mathrm{Rc}} / S_{p, \text { Rc }}$ & \\
\hline & \\
\hline & \\
\hline
\end{tabular}

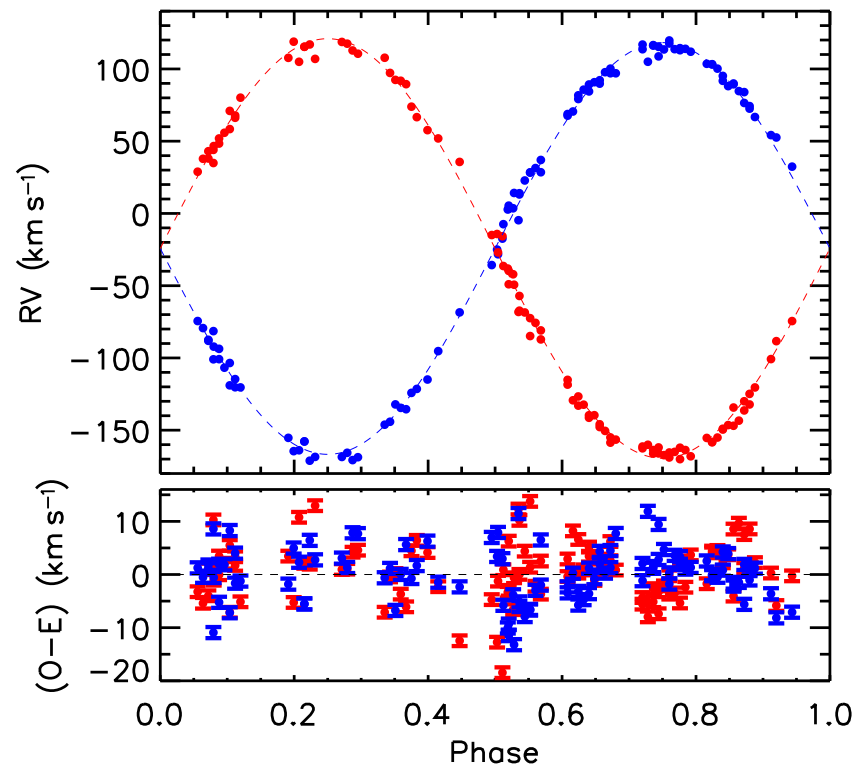

Figure 16. Radial velocities $v_{p}$ (blue) and $v_{s}$ (red) for the primary and secondary stars of GU Boo, as reported by LMR05. We also show the best-fit model as determined from our fitting procedure. In the bottom panel, we show the $(\mathrm{O}-\mathrm{E})$ residuals with respect to the best-fit model.

Software: CPS pipeline (Howard et al. 2010), gatspy (Press \& Rybicki 1989; Vanderplas et al. 2016), lsf_rotate (Gray 1992; Hubeny \& Lanz 2011), MAKEE (http://www.astro.caltech. edu/ tb/makee/), supersmoother (Friedman 1984; Vanderplas \& Willmer 2015).

\section{Appendix \\ Pipeline Validation via Reanalysis of GU Boo}

One of the first benchmark low-mass eclipsing binaries identified and rigorously characterized was GU Boo (López-Morales \& 

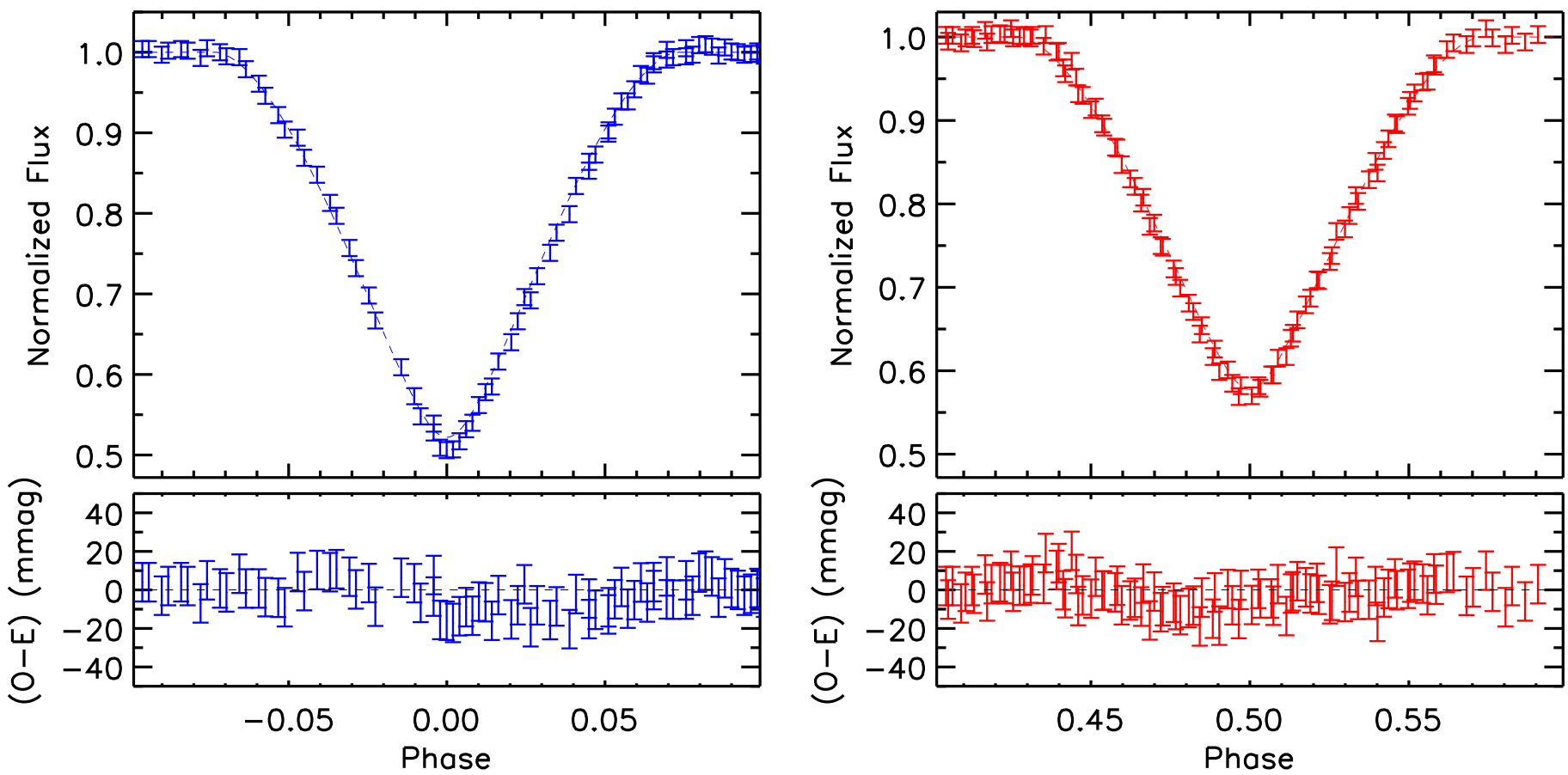

Figure 17. The $R$-band photometry for the primary eclipse (left) and secondary eclipse (right) of GU Boo, as reported by LMR05, along with the best-fit models (dashed lines) and (O-E) residuals (bottom panels).
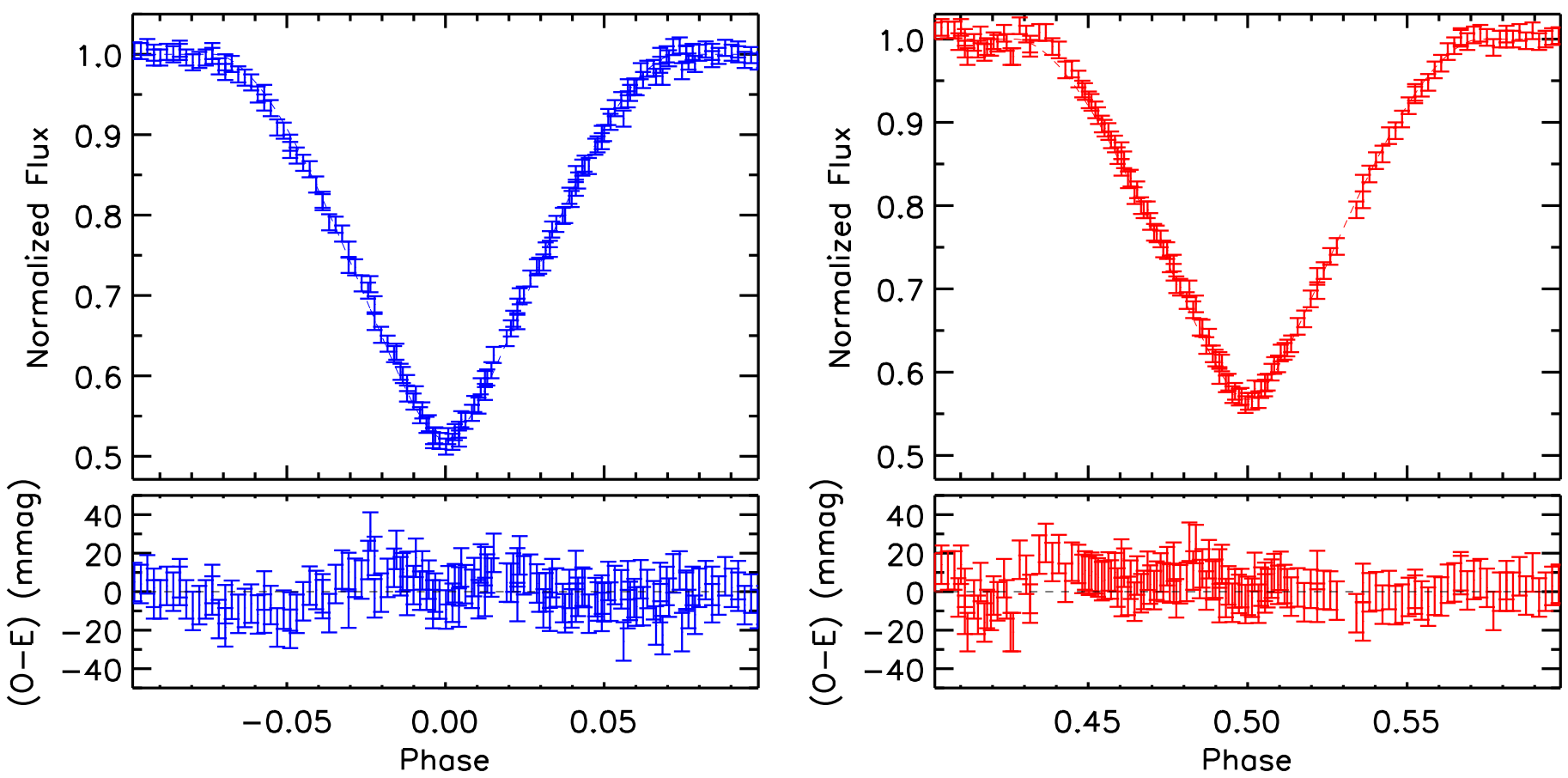

Figure 18. The $I$-band photometry for the primary eclipse (left) and secondary eclipse (right) of GU Boo, as reported by LMR05, along with the best-fit models (dashed lines) and (O-E) residuals (bottom panels).

Ribas 2005; hereafter LMR05), a pair of $\sim 0.6 M_{\odot}(\mathrm{SpT}=\mathrm{M} 0)$ stars in a short-period $(P=0.48$ days) binary system. The RVs and multicolor photometry were published along with the system's discovery and characterization, making it a natural test case for comparing eclipsing binary analysis pipelines. In particular, LMR05 analyzed the system with different choices of software (the 2003 version of the Wilson-Devinney code; Wilson \&
Devinney 1971), convergence algorithm (the standard WilsonDevinney method of differential corrections), treatment of the atmospheres (via Kurucz models; e.g., Kurucz 1979), limb darkening (a square-root law), and treatment of spots (by modeling a large spot on each star).

To test the fidelity of our own pipeline, we reanalyzed the RVs and optical photometry of GU Boo using the parts of 

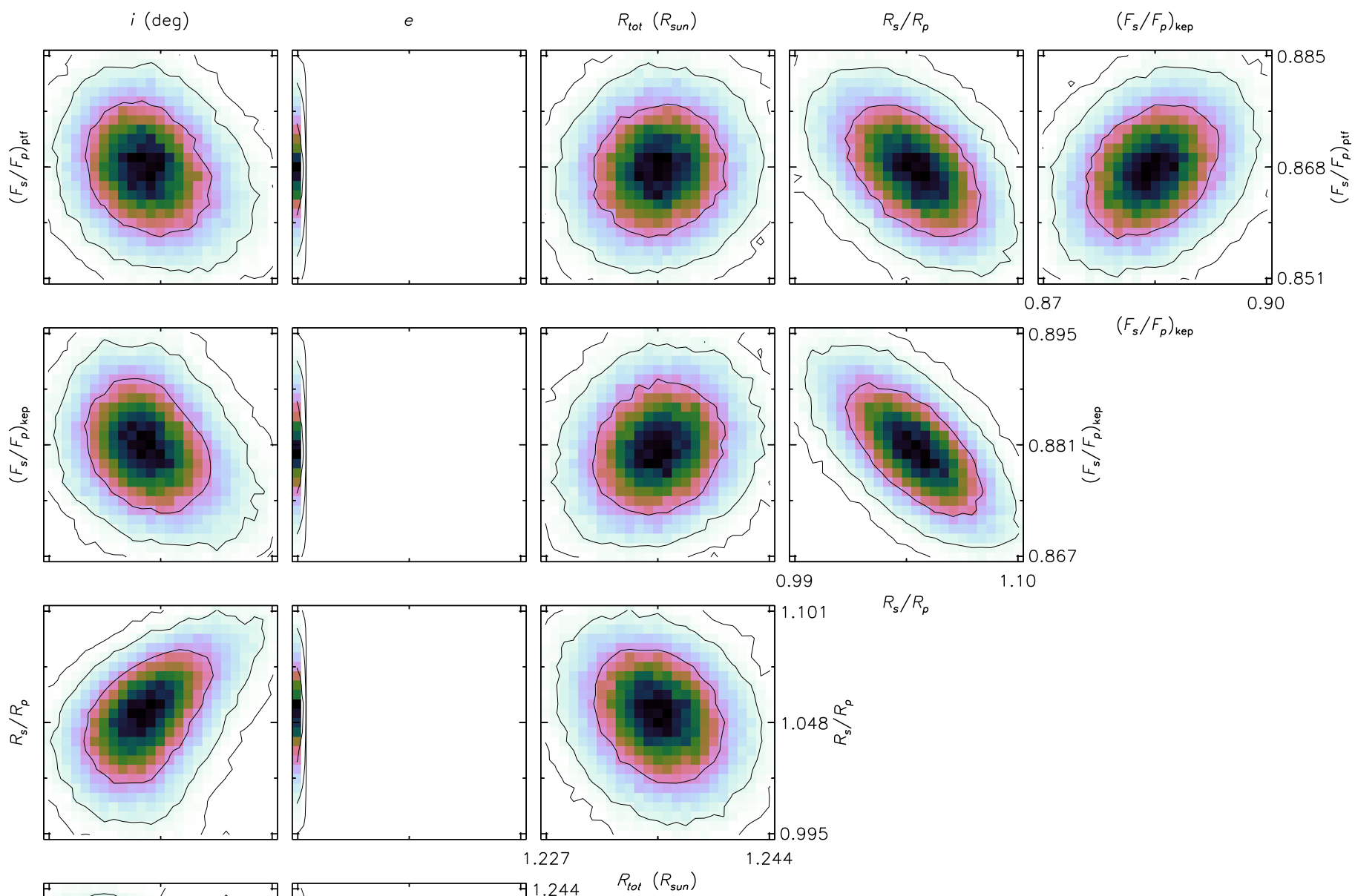

$R_{s} / R_{p}$
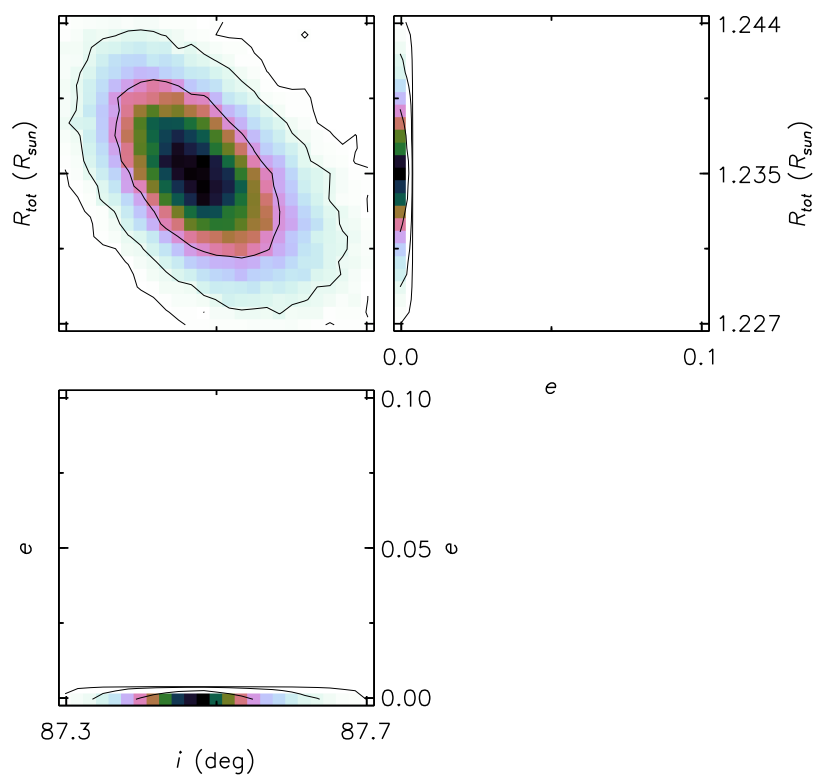

Figure 19. Two-dimensional marginalized posterior distributions for the six fit parameters in our MCMC analysis of GU Boo that are most likely to show astrophysically important covariances. There are moderate covariances between the radius ratio, radius sum, and system inclination (column 1, rows 3 and 4 ), as well as between the radius ratio and the surface brightness ratios (column 4, row 2 and column 5, row 1).

our pipeline for which appropriate data were available. We specifically fit the individual component RVs and the $R_{C}$ and $I_{C}$ light curves but did not attempt to apply any spectroscopic prior. We adopted the LMR05 assumption of component temperatures of $T_{P}=3920$ and $T_{S}=3810 \mathrm{~K}$ and quadratic limb-darkening coefficients of $\gamma_{1, P, R}=0.2516, \quad \gamma_{2, P, R}=$ $0.3528, \quad \gamma_{1, S, R}=0.2562, \quad \gamma_{2, S, R}=0.4203, \quad \gamma_{1, P, I}=0.4558$, $\gamma_{2, P, I}=0.3528, \gamma_{1, S, I}=0.4763$, and $\gamma_{2, S, I}=0.3829$. To rectify the secular influence of spots on the light curve, we fit and divided the phased light curves for near-eclipse observations (within $\Delta \phi<0.15$ ) with quadratic polynomials; we otherwise did not include spot modeling. We fixed the orbital eccentricity to zero and therefore did not fit $e$ or $\omega$. All other parameters were allowed to float, in analogy with our analysis of 
PTFEB132.707+19.810. We explored the posterior parameter distributions for this system with the MCMC pipeline described in Section 3.2, computing 20 simultaneous chains for a total length of $1.1 \times 10^{5}$ steps per chain, omitting the first $10^{4}$ steps of each chain to allow for random dispersal from the (common) initial starting point. We report the resulting system parameters in Table 8 and plot the resulting RV curves (Figure 16), light curves (Figures 17 and 18), and corner plot (Figure 19).

We find that our inferred component masses and the orbital parameters constrained by the RVs are indistinguishable from those of LMR05, as should be expected from fitting the same data with well-understood Newtonian dynamics. We do find modest differences in the parameters set by the light curves (stellar radii and system inclination), but our best-fit values agree with the values reported by LMR05 to within the mutual $1 \sigma$ confidence interval. They found a system inclination of $i=87^{\circ} .6 \pm 0.2$, while our analysis finds a best-fit value of $i=87^{\circ} .46 \pm 0.08(0.75 \sigma$ lower). Similarly, they found stellar radii of $R_{P}=0.623 \pm 0.016$ and $R_{S}=0.620 \pm 0.020 R_{\odot}$, while we found radii of $R_{P}=0.602 \pm 0.007$ and $R_{S}=$ $0.633 \pm 0.006 R_{\odot}$. We found that the sum of the radii was very well constrained $\left(R_{\mathrm{tot}}=1.235 \pm 0.003 R_{\odot}\right)$, in good agreement with their sum $\left(R_{\mathrm{tot}}=1.243 \pm 0.026 R_{\odot}\right)$, which confirms that the anticorrelated discrepancies are due to the well-known difficulty of measuring the ratio of radii in eclipsing binary systems.

We see evidence of red (temporally correlated) noise in the light curves during eclipses, which we attribute to variations in surface brightness on the occulted star due to the presence of spots. In particular, the $R$-band light curve shows a clear asymmetry in the primary eclipse that might indicate a higher spot coverage fraction on the ingress side of the occulted primary star than on the egress side. This asymmetry qualitatively explains the location of the large spot that LMR05 added to the primary star in their system model.

In summary, these results suggest that even given the many methodological differences and absence of spot modeling, our pipeline yields radius measurements that are consistent with previous analysis pipelines to within $<2 \%-3 \%$.

\section{ORCID iDs}

Adam L. Kraus (1) https://orcid.org/0000-0001-9811-568X Stephanie T. Douglas (1ㄴ https://orcid.org/0000-0001-7371-2832 Andrew W. Mann (1) https://orcid.org/0000-0003-3654-1602 Marcel A. Agüeros (i) https://orcid.org/0000-0001-7077-3664 Nicholas M. Law (1) https://orcid.org/0000-0001-9380-6457 Kevin R. Covey (ㄴ) https://orcid.org/0000-0001-6914-7797 Gregory A. Feiden (1) https://orcid.org/0000-0002-2012-7215 Aaron C. Rizzuto @i https://orcid.org/0000-0001-9982-1332 Andrew W. Howard (ㄱ) https://orcid.org/0000-0001-

8638-0320

Howard Isaacson (1) https://orcid.org/0000-0002-0531-1073 Guillermo Torres (1) https://orcid.org/0000-0002-5286-0251 Gaspar Bakos iㅏ https://orcid.org/0000-0001-7204-6727

\section{References}

Adams, J. D., Stauffer, J. R., Skrutskie, M. F., et al. 2002, AJ, 124, 1570 Agüeros, M. A., Covey, K. R., Lemonias, J. J., et al. 2011, ApJ, 740, 110 Ahn, C. P., Alexandroff, R., Allende Prieto, C., et al. 2012, ApJS, 203, 21 Aigrain, S., Parviainen, H., \& Pope, B. J. S. 2016, MNRAS, 459, 2408 Aldering, G., Adam, G., Antilogus, P., et al. 2002, Proc. SPIE, 4836, 61
Allard, F., Homeier, D., \& Freytag, B. 2011, in ASP Conf. Ser. 448, 16th Cambridge Workshop on Cool Stars, Stellar Systems, and the Sun, ed. C. Johns-Krull, M. K. Browning, \& A. A. West (San Francisco, CA: ASP), 91

Baraffe, I., Homeier, D., Allard, F., \& Chabrier, G. 2015, A\&A, 577, A42

Bastian, N., Covey, K. R., \& Meyer, M. R. 2010, ARA\&A, 48, 339

Bastien, F. A., Stassun, K. G., \& Pepper, J. 2014, ApJL, 788, L9

Benedict, G. F., Henry, T. J., Franz, O. G., et al. 2016, AJ, 152, 141

Boudreault, S., Lodieu, N., Deacon, N. R., \& Hambly, N. C. 2012, MNRAS, 426, 3419

Boyajian, T. S., von Braun, K., van Belle, G., et al. 2012, ApJ, 757, 112

Brandt, T. D., \& Huang, C. X. 2015, ApJ, 807, 24

Casagrande, L., Flynn, C., \& Bessell, M. 2008, MNRAS, 389, 585

Chabrier, G., Gallardo, J., \& Baraffe, I. 2007, A\&A, 472, L17

Claret, A., \& Bloemen, S. 2011, A\&A, 529, A75

Claret, A., Hauschildt, P. H., \& Witte, S. 2012, A\&A, 546, A14

Clausen, J. V., Bruntt, H., Claret, A., et al. 2009, A\&A, 502, 253

Cody, A. M., Stauffer, J., Baglin, A., et al. 2014, AJ, 147, 82

Cohen, M., Wheaton, W. A., \& Megeath, S. T. 2003, AJ, 126, 1090

Cushing, M. C., Vacca, W. D., \& Rayner, J. T. 2004, PASP, 116, 362

Cutri, R. M., Skrutskie, M. F., van Dyk, S., et al. 2003, 2MASS All Sky Catalog of Point Sources (Pasadena, CA: NASA/IPAC Infrared Science Archive)

Cutri, R. M., et al. 2013, yCat, 2328, 0

Czekala, I., Andrews, S. M., Mandel, K. S., Hogg, D. W., \& Green, G. M. 2015, ApJ, 812, 128

David, T. J., Conroy, K. E., Hillenbrand, L. A., et al. 2016, AJ, 151, 112

David, T. J., Stauffer, J., Hillenbrand, L. A., et al. 2015, ApJ, 814, 62

Delfosse, X., Forveille, T., Ségransan, D., et al. 2000, A\&A, 364, 217

Delorme, P., Collier Cameron, A., Hebb, L., et al. 2011, MNRAS, 413, 2218

Dittmann, J. A., Irwin, J. M., Charbonneau, D., et al. 2017, ApJ, 836, 124

Dotter, A., Chaboyer, B., Jevremović, D., et al. 2008, ApJS, 178, 89

Douglas, S. T., Agüeros, M. A., Covey, K. R., et al. 2014, ApJ, 795, 161

Douglas, S. T., Agüeros, M. A., Covey, K. R., et al. 2016, ApJ, 822, 47

Douglas, S. T., Agüeros, M. A., Covey, K. R., \& Kraus, A. L. 2017, arXiv: 1704.04507

Eastman, J., Gaudi, B. S., \& Agol, E. 2013, PASP, 125, 83

Feiden, G. A., \& Chaboyer, B. 2014a, ApJ, 789, 53

Feiden, G. A., \& Chaboyer, B. 2014b, A\&A, 571, A70

Friedman, J. H. 1984, A Variable Span Scatterplot Smoother, Technical Report 5 (Laboratory for Computational Statistics, Stanford Univ.)

Fukugita, M., Ichikawa, T., Gunn, J. E., et al. 1996, AJ, 111, 1748

Gaidos, E., \& Mann, A. W. 2012, arXiv:1211.2279

Gillen, E., Hillenbrand, L. A., David, T. J., et al. 2017, arXiv:1706.03084

Gray, D. F. 1992, The Observation and Analysis of Stellar Photospheres (Cambridge: Cambridge Univ. Press)

Green, D. A. 2011, BASI, 39, 289

Harrison, T. E., Coughlin, J. L., Ule, N. M., \& López-Morales, M. 2012, AJ, 143,4

Howard, A. W., Johnson, J. A., Marcy, G. W., et al. 2010, ApJ, 721, 1467

Howell, S. B., Sobeck, C., Haas, M., et al. 2014, PASP, 126, 398

Hubeny, I., \& Lanz, T. 2011, Synspec: General Spectrum Synthesis Program, Astrophysics Source Code Library, ascl:1109.022

Huber, D., Bryson, S. T., Haas, M. R., et al. 2016, ApJS, 224, 2

Iglesias-Marzoa, R., López-Morales, M., Arévalo, M. J., Coughlin, J. L., \& Lázaro, C. 2017, A\&A, 600, A55

Irwin, J. M., Quinn, S. N., Berta, Z. K., et al. 2011, ApJ, 742, 123

Jackson, R., \& Jeffries, R. 2014, MNRAS, 441, 2111

Kovács, G., Zucker, S., \& Mazeh, T. 2002, A\&A, 391, 369

Kraus, A. L., Cody, A. M., Covey, K. R., et al. 2015, ApJ, 807, 3

Kraus, A. L., \& Hillenbrand, L. A. 2007, AJ, 134, 2340

Kraus, A. L., Shkolnik, E. L., Allers, K. N., \& Liu, M. C. 2014, AJ, 147, 146

Kraus, A. L., Tucker, R. A., Thompson, M. I., Craine, E. R., \& Hillenbrand, L. A. 2011, ApJ, 728, 48

Kurucz, R. L. 1979, ApJS, 40, 1

Lantz, B., Aldering, G., Antilogus, P., et al. 2004, Proc. SPIE, 5249, 146

Law, N. M., Kraus, A. L., Street, R., et al. 2012, ApJ, 757, 133

Law, N. M., Kraus, A. L., Street, R. R., et al. 2011, in ASP Conf. Ser. 448, 16th Cambridge Workshop on Cool Stars, Stellar Systems, and the Sun, ed. C. Johns-Krull, M. K. Browning, \& A. A. West (San Francisco, CA: ASP), 1367

Law, N. M., Kulkarni, S. R., Dekany, R. G., et al. 2009, PASP, 121, 1395 López-Morales, M. 2007, ApJ, 660, 732

López-Morales, M., \& Ribas, I. 2005, ApJ, 631, 1120

Mandel, K., \& Agol, E. 2002, ApJL, 580, L171 
Mann, A. W., Feiden, G. A., Gaidos, E., Boyajian, T., \& von Braun, K. 2015, ApJ, 804, 64

Mann, A. W., Gaidos, E., \& Ansdell, M. 2013a, ApJ, 779, 188

Mann, A. W., Gaidos, E., Kraus, A., \& Hilton, E. J. 2013b, ApJ, 770, 43

Mann, A. W., Gaidos, E., Vanderburg, A., et al. 2017, AJ, 153, 64

Mann, A. W., \& von Braun, K. 2015, PASP, 127, 102

Massey, P., Strobel, K., Barnes, J. V., \& Anderson, E. 1988, ApJ, 328, 315

McQuillan, A., Mazeh, T., \& Aigrain, S. 2013, ApJL, 775, L11

Mermilliod, J.-C., \& Mayor, M. 1999, A\&A, 352, 479

Morales, J. C., Ribas, I., Jordi, C., et al. 2009, ApJ, 691, 1400

Mullan, D., \& MacDonald, J. 2001, ApJ, 559, 353

MacDonald, J., \& Mullan, J. 2014, ApJ, 787, 70

Newton, E. R., Irwin, J., Charbonneau, D., et al. 2016, ApJ, 821, 93

Ofek, E. O., Laher, R., Law, N., et al. 2012, PASP, 124, 62

Orosz, J. A., \& Hauschildt, P. H. 2000, A\&A, 364, 265

Popper, D. M. 1997, AJ, 114, 1195

Press, W. H., \& Rybicki, G. B. 1989, ApJ, 338, 277

Rahmer, G., Smith, R., Velur, V., et al. 2008, Proc. SPIE, 7014, 70144Y

Rajpurohit, A. S., Reylé, C., Allard, F., et al. 2013, A\&A, 556, A15

Rayner, J. T., Toomey, D. W., Onaka, P. M., et al. 2003, PASP, 115, 362

Rebull, L. M., Stauffer, J. R., Bouvier, J., et al. 2016, arXiv:1606.00052

Rebull, L. M., Stauffer, J. R., Hillenbrand, L. A., et al. 2017, ApJ, 839, 92

Rucinski, S. 1999, in ASP Conf. Ser. 185, IAU Colloq. 170: Precise Stellar Radial Velocities, ed. J. B. Hearnshaw \& C. D. Scarfe (San Francisco, CA: ASP), 82

Shulyak, D., Reiners, A., Seemann, U., Kochukhov, O., \& Piskunov, N. 2014, A\&A, 563, A35
Shulyak, D., Seifahrt, A., Reiners, A., Kochukhov, O., \& Piskunov, N. 2011, MNRAS, 418, 2548

Sing, D. K. 2010, A\&A, 510, A21

Spruit, H., \& Weiss, A. 1986, A\&A, 166, 167

Somers, G., \& Pinsonneault, M. H. 2015, ApJ, 807, 174

Stassun, K. G., Feiden, G. A., \& Torres, G. 2014, NewAR, 60, 1

Stassun, K., Scholz, A., Dupuy, T., \& Kratter, K. 2014, ApJ, 796, 119

Taylor, B. J. 2006, AJ, 132, 2453

Torres, G., Andersen, J., \& Giménez, A. 2010, A\&ARv, 18, 67

Torres, G., Lacy, C. H., Marschall, L. A., Sheets, H. A., \& Mader, J. A. 2006 ApJ, 640, 1018

Torres, G., Vaz, L. P. R., \& Sandberg Lacy, C. H. 2008, AJ, 136, 2158

Vacca, W. D., Cushing, M. C., \& Rayner, J. T. 2003, PASP, 115, 389

van Leeuwen, F. 2009, A\&A, 497, 209

Vanderburg, A., \& Johnson, J. A. 2014, PASP, 126, 948

Vanderplas, J., Naul, B., Willmer, A., Williams, P., \& Morris, B. M. 2016, Gatspy: General tools for Astronomical Time Series in Python, v0.3, Zenodo, doi: $10.5281 /$ zenodo.47887

Vanderplas, J., \& Willmer, A. 2015, Supersmoother: Efficient Python Implementation of Friedman's SuperSmoother, v0.3.2, Zenodo, doi:10. 5281/zenodo. 28518

Vogt, S. S., Allen, S. L., Bigelow, B. C., et al. 1994, Proc. SPIE, 2198, 362

West, A. A., Hawley, S. L., Bochanski, J. J., et al. 2008, AJ, 135, 785

West, A. A., Morgan, D. P., Bochanski, J. J., et al. 2011, AJ, 141, 97

Wilson, R. E., \& Devinney, E. J. 1971, ApJ, 166, 605

Windmiller, G., Orosz, J. A., \& Etzel, P. B. 2010, ApJ, 712, 1003

Zahn, J. 1977, A\&A, 57, 383 